\title{
Exponential asymptotics and gravity waves
}

\author{
By S. JONATHAN CHAPMAN ${ }^{1}$ \\ AND JEAN-MARC VANDEN-BROECK ${ }^{2}$ \\ ${ }^{1}$ OCIAM, Mathematical Institute, 24-29 St Giles', Oxford OX1 3LB, UK \\ ${ }^{2}$ Department of Mathematics, University of East Anglia, Norwich NR4 7TJ, UK
}

(Received 12 January 2004 and in revised form 5 April 2006)

The problem of irrotational inviscid incompressible free-surface flow is examined in the limit of small Froude number. Since this is a singular perturbation, singularities in the flow field (or its analytic continuation) such as stagnation points, or corners in submerged objects or on rough beds, lead to a divergent asymptotic expansion, with associated Stokes lines. Recent techniques in exponential asymptotics are employed to observe the switching on of exponentially small gravity waves across these Stokes lines.

As a concrete example, the flow over a step is considered. It is found that there are three possible parameter regimes, depending on whether the dimensionless step height is small, of the same order, or large compared to the square of the Froude number. Asymptotic results are derived in each case, and compared with numerical simulations of the full nonlinear problem. The agreement is remarkably good, even at relatively large Froude number. This is in contrast to the alternative analytical theory of small step height, which is accurate only for very small steps.

\section{Introduction}

Free-surface flows over rough surfaces or submerged bodies have been studied for over a century. Early work includes that of Kelvin (1886), who considered the stationary wave pattern caused by finite elevations or depressions in the bed of a stream, and Lamb (1913), who found the two-dimensional velocity potential due to a fixed circular cylinder in a uniform stream, under the assumption that the cylinder is small and the free-surface conditions are linearized.

Several authors have used singularities to model both finite and infinite bodies moving in a free stream, for example Havelock (1927) and Gazdar (1973). Dean (1948) and Ursell (1950) consider a wave incident on a fixed submerged circular cylinder, using a linearized theory of small disturbances. Wilmott (1987) applies the technique of matched asymptotic expansions to the motion of a small body beneath a free surface. All these works rely on a linearization of the free-surface conditions, either in an ad hoc fashion, or due to the disturbing body being small in comparison to its distance from the free surface.

With the advent of the computer, it became possible to retain the full nonlinear free-surface condition using numerical simulation. For example, von Kerczek \& Salvesen (1977) consider numerically the generation of waves on the surface of a stream by an imposed pressure distribution on the surface. Forbes \& Schwartz (1982) consider flow over a semi-circular bump in the bed of a stream of finite depth using a Joukowski transformation on the potential plane to reduce the problem to 
an integro-differential equation. Analytical results are given in the linear case, and numerical results presented using the full nonlinear boundary condition. This work was generalized to a system of two superposed fluids and to an arbitrary topography of the bottom by Belward \& Forbes (1993) and Dias \& Vanden-Broeck (2004). In a similar vein, King \& Bloor (1987) consider the flow over a step by again formulating the problem as a nonlinear integro-differential equation. Analytical results are given for small step height, and numerical results presented for arbitrary steps. This was generalized to flow over a submerged body in King \& Bloor (1989), and to flow over an arbitrary bed topography in King \& Bloor (1990). A numerical method involving isolated sources located outside the flow domain is used to compute potential flows over submerged cylinders in Scullen \& Tuck (1995). Ploughing flows, in which a uniform stream is split by a semi-infinite obstacle are studied numerically in Tuck \& Vanden-Broeck (1998). Various nonlinear free-surface flows generated by submerged sources and sinks were calculated numerically by Tuck \& Vanden-Broeck (1984), Forbes \& Hocking (1990), and Vanden-Broeck \& Keller (1997). These flows model selective withdrawal from reservoirs.

Supercritical flows (in which the Froude number is greater than unity) of a finitedepth fluid with a mat or distributed sink are considered by Koerber \& Forbes (1998, 1999, respectively).

In three dimensions, Tuck \& Scullen (2002) compared full numerical simulations both with an ad hoc linearization of the free-surface condition, and with a consistent thin ship approximation, for submerged spheroids.

In all these works, analytic results are available when the disturbance to the free stream is small, but for more general situations, a numerical simulation is required. In this paper, we aim to extend our knowledge of the generation of gravity waves by a rough bottom or submerged object in a free stream by considering a different asymptotic limit in which analytical progress can be made. Specifically, we consider an arbitrary flow geometry in the limit of small Froude number. In this limit, the dominance of gravity means that the free surface is again flat to leading order, even though the disturbance to the flow by the object is great.

The problem is a singular perturbation problem, and we would therefore expect the formal asymptotic series developed in powers of the Froude number to be divergent. This divergence is associated with the presence of an exponentially small correction to the algebraic expansion, which appears 'beyond all orders' (Dingle 1973).

Kruskal \& Segur (1991) observed that in nonlinear ordinary differential equations, the divergence is caused by a singularity in the analytic continuation of the leadingorder solution, near which the formal asymptotic series breaks down. They formulate an inner problem in the vicinity of the singularity, and find that its solution contains a decaying exponential in the far field, in addition to the terms which match with the algebraic series. This exponential is matched to a solution of the linearized equation away from the singularity to obtain the exponentially small, beyond all orders correction. Kruskal \& Segur (1991) solve the inner problem numerically (observing the exponential along a ray on which the algebraic terms vanished), but other authors have since observed that the far field of the inner solution can be Borel-summed to obtain the exponential to be matched to the outer solution (see, for example, Combescot et al. 1988; Hakim 1991).

A similar method is formulated in Chapman, King \& Adams (1998), Chapman (1999) and Chapman \& Vanden-Broeck (2002), where the algebraic asymptotic expansion (away from the singularity) is optimally truncated (that is, truncated at its least term), which results in an exponentially small error between the truncated 
series and the exact solution. This allows the exponentially small corrections to be observed switching on across Stokes lines by smoothing the Stokes discontinuity, and is a generalization of the smoothing of Stokes discontinuities in linear ordinary differential equations discovered by Berry (1989).

The exponentially small correction terms which appear in the present problem correspond to gravity waves on the free surface of exponentially small amplitude. We will develop a procedure to determine the region of the free surface they occupy, as well as their magnitude, for general flow geometries. We note that exponentially small gravity-capillary waves have been studied in the context of the Korteweg-de Vries equation in Akylas \& Yang (1995), Byatt-Smith (1991), Grimshaw \& Joshi (1995) and Pomeau, Ramani \& Grammaticos (1988).

The rest of the paper is organized as follows. In $\S 2$, we formulate the problem mathematically. In $\S \S 3$ and 4, we apply the procedure developed in Chapman et al. (1998). In $\S 3$, we naively expand the solution as a formal power series in the Froude number and find the form of the late terms in this series. This enables us, in $\S 4$, to truncate the algebraic series optimally, and to observe the subdominant exponentials being switched on across Stokes lines.

In $\S 5$, we consider as a test problem the flow over a step, considered previously by King \& Bloor (1987). The analytical results in King \& Bloor (1987) are for small step height, but we find that the small-step-height limit of our result does not agree with the small-Froude-number limit of their result: the two limits do not commute. In Appendix A, we apply our procedure to the limiting problem of small step height (thus applying the limits in reverse order), and show that it agrees with the result of King \& Bloor (1987). In Appendix B, we consider the canonical scaling in which the Froude number and step height both tend to zero at the appropriate rate.

In $\S 6$, we compare the results of $\S 5$, Appendix B and King \& Bloor (1987) with a direct numerical simulation of the full problem. We find that a uniform approximation generated from the results of $\S 5$ and Appendix B is remarkably accurate, even for relatively large Froude numbers. This is in contrast to the small-step-height approximation, which is good only for very small steps (at moderate Froude numbers).

Finally, in $\S 7$, we present our conclusions.

\section{Formulation of the problem}

We consider a general steady, incompressible, irrotational, inviscid flow in the presence of gravity with small Froude number. The fluid velocity $\boldsymbol{u}=(u, v)=\nabla \phi$, where the velocity potential $\phi$ satisfies

$$
\nabla^{2} \phi=0 .
$$

On all boundaries we have the kinematic condition

$$
\frac{\partial \phi}{\partial n}=0
$$

while on the free boundary we also have the dynamic condition, which from Bernoulli's equation is

$$
\epsilon\left(\frac{1}{2}|\nabla \phi|^{2}-\frac{1}{2}\right)+y=0
$$

where $\epsilon=U^{2} / g L$ is the square of the Froude number, and we have non-dimensionalized the fluid velocity $\nabla \phi$ with the flow at infinity, $U$, and length with a typical length $L$ which will depend upon the exact geometry being considered. For flow in a 
channel, $L$ will be a typical channel depth, while for flow over a submerged object, $L$ will be the depth of the object.

As usual, we define the complex potential by $w=\phi+\mathrm{i} \psi$, where $\psi$ is the streamfunction (with $\psi$ chosen to be zero on the free boundary), and we let $z=x+i y$. Since $w$ is then an analytic function of $z$, the map $z \rightarrow w$ is a conformal transformation of the flow region to a region of the potential plane.

It is convenient to work in terms of the complex velocity $\mathrm{d} w / \mathrm{d} z=u-\mathrm{i} v$, which may be written as $q \mathrm{e}^{-\mathrm{i} \theta}$, where $\theta$ is the angle the streamlines make with the $x$-axis. Then

$$
\frac{\mathrm{d} x}{\mathrm{~d} s}=\cos \theta, \quad \frac{\mathrm{d} y}{\mathrm{~d} s}=\sin \theta,
$$

where $s$ is arclength, so that differentiating (2.1) gives

$$
\epsilon q \frac{\mathrm{d} q}{\mathrm{~d} s}=-\sin \theta,
$$

on the free boundary. Noting that

$$
\frac{\mathrm{d} q}{\mathrm{~d} s}=\frac{\mathrm{d} q}{\mathrm{~d} \phi} \frac{\partial \phi}{\partial s}=q \frac{\mathrm{d} q}{\mathrm{~d} \phi}
$$

in the potential plane this is

$$
\epsilon q^{2} \frac{\mathrm{d} q}{\mathrm{~d} \phi}=-\sin \theta .
$$

A second transformation $w \rightarrow \zeta$ is often used to map the flow region in the potential plane to the upper half- $\zeta$-plane. Then the analyticity of $q \mathrm{e}^{-\mathrm{i} \theta}$ in the upper half-plane implies

$$
\log q=-\frac{1}{\pi} f_{-\infty}^{\infty} \frac{\theta\left(\xi^{\prime}\right) \mathrm{d} \xi^{\prime}}{\xi^{\prime}-\xi},
$$

where $\zeta=\xi+\mathrm{i} \eta$.

Now in the course of our analysis we will need to complexify the free boundary, i.e. complexify $x, y, s, \phi, q$ and $\theta$. Since we are dealing with complex variables $z, \zeta$ and $w$ anyway this can lead to some confusion. The complexification of $\phi$ is simply $w$, that of $\xi$ is simply $\zeta$. The only difference between the complexified free boundary and the flow region is that on the complexified free boundary $q$ and $\theta$ are not real, even though $q \mathrm{e}^{-\mathrm{i} \theta}$ is still equal to $\mathrm{d} w / \mathrm{d} z$.

Analytically continuing (2.4) into the upper half- $\xi$-plane gives

$$
\log q-\mathrm{i} \theta=-\frac{1}{\pi} \int_{-\infty}^{\infty} \frac{\theta\left(\zeta^{\prime}\right) \mathrm{d} \zeta^{\prime}}{\zeta^{\prime}-\zeta},
$$

where we have replaced $\xi$ by $\zeta$ to avoid confusion. Similarly, we will write (2.3) for complex $\phi$ by replacing $\phi$ by $w$ so that

$$
\epsilon q^{2} \frac{\mathrm{d} q}{\mathrm{~d} w}=-\sin \theta .
$$

Thus our problem is (2.5) and (2.6), together with the conformal map $w \rightarrow \zeta$ which depends on the geometry of the particular problem we are considering. We can perform much of our analysis without knowing the precise form of this map. Thus, in the next two sections we leave it unspecified, so that the results are applicable to a general flow problem. Then, in $\S 5$, we apply these results to the particular example of flow over a step. 


\section{Asymptotic expansion for small Froude number}

We will solve (2.5) and (2.6) asymptotically as $\epsilon \rightarrow 0$, with the particular goal of identifying the exponentially small terms beyond all orders, which correspond to the gravity waves on the free surface. The procedure developed in Chapman et al. (1998) is as follows. The first step is to determine the algebraic expansion in powers of $\epsilon$. The presence of exponentially small correction terms means that this expansion is divergent, and so must be truncated. If we truncate it after $N$ terms, where $N$ is fixed, then the error is $O\left(\epsilon^{N}\right)$ as $\epsilon \rightarrow 0$. However, if we truncate it at its least term (that is, we truncate it optimally), then the error is exponentially small in $\epsilon$ (note that the number of terms in the optimal truncation depends on $\epsilon$, so that $N$ increases as $\epsilon$ decreases). By examining the behaviour of the remainder after optimal truncation, we are able to observe directly exponentially small correction terms being switched on across Stokes lines.

Because the number of terms in the optimally truncated series increases as $\epsilon$ decreases, to determine the optimal truncation point and the remainder after optimal truncation we do not need to know the full asymptotic expansion in powers of $\epsilon$, but only the late terms in the expansion, that is, the coefficient of $\epsilon^{n}$ for large $n$.

\subsection{Late terms in the regular asymptotic expansion}

We expand in powers of $\epsilon$ as

$$
\theta=\sum_{n=0}^{\infty} \epsilon^{n} \theta_{n}, \quad q=\sum_{n=0}^{\infty} \epsilon^{n} q_{n} .
$$

Then, at leading order,

$$
\begin{aligned}
\theta_{0} & =0 \quad \text { on the free boundary, } \\
\log q_{0} & =-\frac{1}{\pi} f_{-\infty}^{\infty} \frac{\theta_{0}\left(\xi^{\prime}\right) \mathrm{d} \xi^{\prime}}{\xi^{\prime}-\xi} .
\end{aligned}
$$

Note that the integral in (3.3) corresponds to integrating over the boundary of the fluid domain, and will therefore contain sections corresponding to fixed boundaries (on which $\theta$ is known), and a section corresponding to the free boundary (on which (3.2) holds); typically the free boundary will correspond to $0<\xi<\infty$ (see $\S 5$, and in particular equation (5.2), for example). At first order, we have

$$
\begin{aligned}
q_{0}^{2} \frac{\mathrm{d} q_{0}}{\mathrm{~d} w} & =-\theta_{1} \quad \text { on the free boundary, } \\
\frac{q_{1}}{q_{0}}-\mathrm{i} \theta_{1} & =-\frac{1}{\pi} \int_{-\infty}^{\infty} \frac{\theta_{1}\left(\zeta^{\prime}\right) \mathrm{d} \zeta^{\prime}}{\zeta^{\prime}-\zeta},
\end{aligned}
$$

where we have replaced (2.4) with (2.5), and in general

$$
\begin{gathered}
q_{0}^{2} \frac{\mathrm{d} q_{n-1}}{\mathrm{~d} w}+2 q_{0} q_{1} \frac{\mathrm{d} q_{n-2}}{\mathrm{~d} w}+2 q_{0} q_{n-1} \frac{\mathrm{d} q_{0}}{\mathrm{~d} w}+\cdots=-\theta_{n} \cos \theta_{0}+\theta_{1} \theta_{n-1} \sin \theta_{0}+\cdots \quad(n \geqslant 3), \\
\frac{q_{n}}{q_{0}}-\frac{q_{n-1} q_{1}}{q_{0}^{2}}+\cdots-\mathrm{i} \theta_{n}=-\frac{1}{\pi} \int_{-\infty}^{\infty} \frac{\theta_{n}\left(\zeta^{\prime}\right) \mathrm{d} \zeta^{\prime}}{\zeta^{\prime}-\zeta} \quad(n \geqslant 2) .
\end{gathered}
$$

The terms on the left-hand side of (3.6) come from expanding the product of three series $q^{2} \mathrm{~d} q / \mathrm{d} w$, those on the right-hand side of (3.6) come from expanding $-\sin \theta$, while those on the left-hand side of (3.7) come from expanding $\log q$. In each case, we have listed the terms which we will see are dominant for large $n$, since as we have 
said, in order to truncate the series (3.1) optimally we need to know the behaviour of the late terms in the expansion. Thus, we aim to solve (3.6) and (3.7) in the limit as $n \rightarrow \infty$.

Now in the leading-order problem, $q_{0}$ may have singularities as a function of $w$ (through the conformal map $\zeta$ ). However, all higher-order problems are linear and can therefore introduce no new singularities in $q$. Thus, the singular points of $q_{n}(w)$ must be the same as those of $q_{0}(w)$ for all $n$. The singular perturbation in (2.6) means that to determine each successive term in the expansion we differentiate the previous term. Thus, if $q_{n}$ has a singularity of the form $1 /\left(w-w_{0}\right)^{n}$, then $q_{n+1}$ will have a singularity of the form $n /\left(w-w_{0}\right)^{n+1}$. Therefore, as $n \rightarrow \infty$, we expect the asymptotic expansions (3.1) to exhibit factorial/power divergence, so that we make the ansatz

$$
\theta_{n} \sim \frac{\Theta \Gamma(n+\gamma)}{\chi^{n+\gamma}}, \quad q_{n} \sim \frac{Q \Gamma(n+\gamma)}{\chi^{n+\gamma}} \quad \text { as } n \rightarrow \infty,
$$

where $\Theta, Q, \chi$ and $\gamma$ may be functions of $w$, but are independent of $n$. This is similar to a WKBJ ansatz, with $\chi$ the phase, and $\Theta$ and $Q$ the amplitudes. We will find that the terms on the left-hand side of (3.7) exponentially dominate those on the right-hand side for large $n$, so that

$$
\theta_{n} \sim-\frac{\mathrm{i} q_{n}}{q_{0}}+\frac{\mathrm{i} q_{1} q_{n-1}}{q_{0}^{2}}+\cdots \quad \text { as } n \rightarrow \infty,
$$

or equivalently

$$
q_{n} \sim \mathrm{i} q_{0} \theta_{n}+\mathrm{i} \theta_{n-1} q_{1}+\cdots \quad \text { as } n \rightarrow \infty .
$$

Then at leading order as $n \rightarrow \infty$ in (3.6), using (3.10) and the ansatz (3.8) we have

$$
\mathrm{i} q_{0}^{3} \frac{\mathrm{d} \chi}{\mathrm{d} w}=1,
$$

so that

$$
\chi=-\mathrm{i} \int \frac{\mathrm{d} w}{q_{0}^{3}} .
$$

Now, since the singularities of $q_{n}$ must be the same as those of $q_{0}, \chi$ must vanish at one of these singularities, at $w=w_{0}$ say. Then

$$
\chi=-\mathrm{i} \int_{w_{0}}^{w} \frac{\mathrm{d} w}{q_{0}^{3}} .
$$

Proceeding to the next order in $n$ in (3.6) we find $\gamma=$ constant, and then at the next order

$$
q_{0}^{2} Q^{\prime}-2 q_{0} q_{1} Q \chi^{\prime}+2 q_{0} q_{0}^{\prime} Q=-\frac{\mathrm{i} q_{1} Q}{q_{0}^{2}}
$$

where $^{\prime} \equiv \mathrm{d} / \mathrm{d} w$, i.e.

$$
\frac{Q^{\prime}}{Q}=-\frac{2 q_{0}^{\prime}}{q_{0}}-\frac{3 \mathrm{i} q_{1}}{q_{0}^{4}}
$$

by (3.11). Hence

$$
\log Q=-2 \log q_{0}-3 \mathrm{i} \int \frac{q_{1}}{q_{0}^{4}} \mathrm{~d} w+\text { constant }
$$


i.e.

$$
Q=\frac{\Lambda}{q_{0}^{2}} \exp \left(-3 \mathrm{i} \int_{w^{*}}^{w} \frac{q_{1}}{q_{0}^{4}} \mathrm{~d} w\right)
$$

where the endpoint of the integration is fixed at $w^{*}$, which may be chosen arbitrarily providing the resulting integral exists (this may preclude choosing $w^{*}=w_{0}$, which would be the most natural choice otherwise); changing the endpoint of the integration simply changes the value of the constant $\Lambda$. By (3.9) and (3.8), the amplitude function for the late terms in $\theta$ is given by

$$
\Theta=-\frac{\Lambda \mathrm{i}}{q_{0}^{3}} \exp \left(-3 \mathrm{i} \int_{w^{*}}^{w} \frac{q_{1}}{q_{0}^{4}} \mathrm{~d} w\right) .
$$

Thus, we have determined the late terms in the expansion of $\theta$ and $q$, up to the (as yet) arbitrary constants $\gamma$ and $\Lambda$. To summarize, we have

$\theta_{n} \sim-\frac{\mathrm{i} \Lambda}{q_{0}^{3}} \exp \left(-3 \mathrm{i} \int_{w^{*}}^{w} \frac{q_{1}}{q_{0}^{4}} \mathrm{~d} w\right) \frac{\Gamma(n+\gamma)}{\chi^{n+\gamma}}, \quad q_{n} \sim \frac{\Lambda}{q_{0}^{2}} \exp \left(-3 \mathrm{i} \int_{w^{*}}^{w} \frac{q_{1}}{q_{0}^{4}} \mathrm{~d} w\right) \frac{\Gamma(n+\gamma)}{\chi^{n+\gamma}}$,

with $\chi$ given by (3.13). To determine $\gamma$, we must examine the order of the singularity in $\theta_{0}$ and $q_{0}$ as $w \rightarrow w_{0}$, and make sure it is consistent with (3.17), which we will do in $\S 3.2$. To determine $\Lambda$, we must match with an inner region in the vicinity of $w_{0}$, which we will do in $\S 3.3$.

To do this matching we need to know the behaviour of (3.17) as $w \rightarrow w_{0}$, which we now determine. We have

$$
\begin{aligned}
q_{1} & =\mathrm{i} q_{0} \theta_{1}-\frac{q_{0}}{\pi} \int_{-\infty}^{\infty} \frac{\theta_{1}\left(\xi^{\prime}\right) \mathrm{d} \xi^{\prime}}{\xi^{\prime}-\xi} \\
& =-\mathrm{i} q_{0}^{3} \frac{\mathrm{d} q_{0}}{\mathrm{~d} w}-\frac{q_{0}}{\pi} \int_{-\infty}^{\infty} \frac{\theta_{1}\left(\xi^{\prime}\right) \mathrm{d} \xi^{\prime}}{\xi^{\prime}-\xi}
\end{aligned}
$$

Hence,

$$
\begin{aligned}
\exp \left(-3 \mathrm{i} \int_{w^{*}}^{w} \frac{q_{1}}{q_{0}^{4}} \mathrm{~d} w\right) & =\exp \left(-3 \int_{w^{*}}^{w} \frac{q_{0}^{\prime}}{q_{0}} \mathrm{~d} w\right) \times \exp \left(3 \mathrm{i} \int_{w^{*}}^{w} \frac{1}{\pi q_{0}^{3}} \int_{-\infty}^{\infty} \frac{\theta_{1}\left(\xi^{\prime}\right) \mathrm{d} \xi^{\prime}}{\xi^{\prime}-\xi} \mathrm{d} w\right) \\
& =\frac{q_{0}^{3}\left(w^{*}\right)}{q_{0}^{3}(w)} \times \exp \left(3 \mathrm{i} \int_{w^{*}}^{w} \frac{1}{\pi q_{0}^{3}} \int_{-\infty}^{\infty} \frac{\theta_{1}\left(\xi^{\prime}\right) \mathrm{d} \xi^{\prime}}{\xi^{\prime}-\xi} \mathrm{d} w\right),
\end{aligned}
$$

since $q_{0} \rightarrow 1$ as $w \rightarrow-\infty$. Thus, as $n \rightarrow \infty$ and $w \rightarrow w_{0}$,

$$
\theta_{n} \sim-\frac{\mathrm{i} \Lambda C}{q_{0}^{6}} \frac{\Gamma(n+\gamma)}{\chi^{n+\gamma}}, \quad q_{n} \sim \frac{\Lambda C}{q_{0}^{5}} \frac{\Gamma(n+\gamma)}{\chi^{n+\gamma}},
$$

where

$$
C=q_{0}^{3}\left(w^{*}\right) \exp \left(3 \mathrm{i} \int_{w^{*}}^{w_{0}} \frac{1}{\pi q_{0}^{3}} \int_{-\infty}^{\infty} \frac{\theta_{1}\left(\xi^{\prime}\right) \mathrm{d} \xi^{\prime}}{\xi^{\prime}-\xi} \mathrm{d} w\right)
$$

\subsection{Inner limit of $q_{0}$}

To determine $\gamma$, we must examine the order of the singularity in $q_{0}$ as $w \rightarrow w_{0}$. This will depend on the type of singularity in the flow field. Typical singularities include stagnation points, sources, sinks and flow around the corner of an obstacle.

For stagnation points and flow around corners, locally in the vicinity of the singularity we have $w-w_{0}=$ const $\times\left(z-z_{0}\right)^{k}$ for some $k \neq 1$. For a stagnation point 
generically $k=2$, whereas for flow around a corner $k=\pi / \beta$ where $\beta$ is the interior (i.e. in the fluid) angle of the corner. Then, for the corresponding singularity on the analytic continuation of the free boundary

$$
\frac{\mathrm{d} w}{\mathrm{~d} z}=q_{0} \mathrm{e}^{-\mathrm{i} \theta_{0}}=q_{0}=\text { const } \times\left(z-z_{0}\right)^{k-1}=\text { const } \times\left(w-w_{0}\right)^{(k-1) / k},
$$

remembering that $\theta_{0}=0$. Thus, $q_{0} \sim c\left(w-w_{0}\right)^{\alpha}$ where $\alpha=(k-1) / k$ and $c$ is constant, and we have

$$
\chi \sim-\frac{\mathrm{i}\left(w-w_{0}\right)^{1-3 \alpha}}{c^{3}(1-3 \alpha)} .
$$

Remembering that we need $\chi$ to vanish at $w=w_{0}$, we see that we require $\alpha<1 / 3$. Only those singularities with $\alpha<1 / 3$ will generate Stokes lines and their associated exponentially small correction terms. In particular, this means that Stokes lines (and gravity waves) are not generated by stagnation points, and will only be generated by corners with in-the-fluid angles greater than $2 \pi / 3$ (unlike the case of capillary waves studied in Chapman \& Vanden-Broeck 2002). At singularities with $\alpha \geqslant 1 / 3$, the phase $\chi \rightarrow \infty$. Although not generated by such singularities (which correspond to the point at infinity in the standard examples from second-order linear ordinary differential equations) Stokes lines will often end at them; they are discussed in more detail in Chapman \& King (2003).

Now, for the asymptotic behaviour (3.21) to be consistent with (3.18) we require that

$$
(1-3 \alpha) \gamma+5 \alpha=-\alpha,
$$

i.e.

$$
\gamma=-\frac{6 \alpha}{1-3 \alpha}
$$

Finally, the alternative to $w-w_{0}$ being a power of $z$ locally is to have $w-$ $w_{0}=$ const $\times \log \left(z-z_{0}\right)$, corresponding to a source or sink. In that case,

$$
\frac{\mathrm{d} w}{\mathrm{~d} z}=q_{0} \exp \left(-\mathrm{i} \theta_{0}\right)=q_{0}=\text { const } \times\left(z-z_{0}\right)^{-1}=\text { const } \times \exp \left(-\left(w-w_{0}\right)\right),
$$

and there is no singularity in $q_{0}$.

\subsection{Inner expansion in the vicinity of the complex singularity}

To determine $\Lambda$, we must match with an inner solution in the vicinity of the singularity in the complex plane. Now in the vicinity of the complex singularity $w_{0}, \log q-\mathrm{i} \theta$ is equal to the (regular) expansion of the right-hand side of (2.5), which is evaluated on the real axis, and therefore involves the outer expansion of $q$ and $\theta$, away from the singularity. However, we know that inserting the expansions for $q$ and $\theta$ into the right-hand side simply gives the outer expansion of $\log q-\mathrm{i} \theta$. The right-hand side of this equation in the inner region is therefore simply the inner limit of $\log q-\mathrm{i} \theta$. Hence, since $\theta_{0}=0$ and

$$
q_{0} \sim c\left(w-w_{0}\right)^{\alpha},
$$

in the vicinity of $w_{0}$, we have

$$
\begin{aligned}
q \mathrm{e}^{-\mathrm{i} \theta} & =c\left(w-w_{0}\right)^{\alpha}+\cdots, \\
\epsilon q^{2} \frac{\mathrm{d} q}{\mathrm{~d} w} & =-\sin \theta .
\end{aligned}
$$


Motivated by (3.21) we define the inner variable $\eta$ by $w=w_{0}+\epsilon^{1 /(1-3 \alpha)} \eta$ (remembering that $\alpha<1 / 3)$, and set $q=\epsilon^{\alpha /(1-3 \alpha)} c \eta^{\alpha} \bar{q}$. Then, using (3.23) in (3.24) gives

$$
2 \mathrm{i} c^{3} \bar{q}^{2}\left(\alpha \eta^{3 \alpha-1} \bar{q}+\eta^{3 \alpha} \frac{\mathrm{d} \bar{q}}{\mathrm{~d} \eta}\right)=-\bar{q}+\frac{1}{\bar{q}},
$$

Writing

$$
z=\frac{\mathrm{i} \eta^{1-3 \alpha}}{c^{3}(1-3 \alpha)}
$$

gives

$$
-\frac{2 \alpha \bar{q}^{3}}{z(1-3 \alpha)}-2 \bar{q}^{2} \frac{\mathrm{d} \bar{q}}{\mathrm{~d} z}=-\bar{q}+\frac{1}{\bar{q}},
$$

To determine $\Lambda$ we must match the inner limit of (3.18) with the outer limit of (3.26). The easiest way to do this is to write $\phi=\bar{q}^{2}$ giving

$$
-\frac{2 \alpha \phi^{2}}{z(1-3 \alpha)}-\phi \frac{\mathrm{d} \phi}{\mathrm{d} z}=-\phi+1 \text {. }
$$

We expand $\phi$ as $z \rightarrow \infty$ as

$$
\phi=\sum_{n=0}^{\infty} \frac{(-1)^{n} \phi_{n}}{z^{n}}
$$

where

$$
\begin{aligned}
\phi_{0} & =1, \\
\phi_{n} & =\sum_{m=0}^{n-1}\left(m-\frac{2 \alpha}{1-3 \alpha}\right) \phi_{m} \phi_{n-m-1} .
\end{aligned}
$$

Now the outer limit of the $n$th term in the inner expansion of $q^{2}$

$$
\sim \frac{c^{2} \eta^{2 \alpha} \epsilon^{2 \alpha /(1-3 \alpha)} \phi_{n}}{z^{n}},
$$

while the inner limit of the $n$th term in the outer expansion

$$
\begin{aligned}
\sim 2 \epsilon^{n} q_{0} q_{n} & \sim \frac{2 \Lambda C \epsilon^{n} \Gamma(n+\gamma)}{q_{0}^{4} \chi^{n+\gamma}} \\
& \sim \frac{2 \Lambda C \Gamma(n+\gamma) c^{3 \gamma-4}(1-3 \alpha)^{\gamma} \eta^{2 \alpha} \epsilon^{2 \alpha /(1-3 \alpha)}}{\mathrm{e}^{\mathrm{i} \pi \gamma / 2}(-z)^{n}} .
\end{aligned}
$$

Matching (3.30) with (3.31) gives

$$
\Lambda=\frac{c^{6-3 \gamma} \mathrm{e}^{\mathrm{i} \pi \gamma / 2}}{2 C(1-3 \alpha)^{\gamma}} \lim _{n \rightarrow \infty} \frac{\phi_{n}}{\Gamma(n+\gamma)} .
$$

\section{Stokes line smoothing}

Having determined the late terms of the divergent asymptotic expansion we are in a position to identify the exponentially small terms switched on across Stokes lines. We keep the analysis here brief since the details are very similar to calculations which have appeared elsewhere (Chapman et al. 1998; Chapman 1999; Chapman \& Vanden-Broeck 2002). 
We begin by truncating the expansion after $N$ terms, so that

$$
\theta=\sum_{n=0}^{N-1} \epsilon^{n} \theta_{n}+R_{N}, \quad q=\sum_{n=0}^{N-1} \epsilon^{n} q_{n}+S_{N} .
$$

Then the remainders $S_{N}$ and $R_{N}$ satisfy

$$
\begin{gathered}
\frac{S_{N}}{q_{0}}-\frac{\epsilon q_{1} S_{N}}{q_{0}^{2}}-\frac{\epsilon^{N} q_{1} q_{N-1}}{q_{0}^{2}}+\cdots=\mathrm{i} R_{N} \\
\epsilon q_{0}^{2} S_{N}^{\prime}+2 \epsilon q_{0} q_{0}^{\prime} S_{N}+2 \epsilon^{2} q_{0} q_{1} S_{N}^{\prime}+2 \epsilon^{2} q_{0} q_{1}^{\prime} S_{N}+\epsilon^{2} q_{1} q_{0}^{\prime} S_{N}+\epsilon^{N} \theta_{N}+\cdots=R_{N}+\cdots
\end{gathered}
$$

where the omitted terms are lower order as $N \rightarrow \infty$ and $\epsilon \rightarrow 0$. The homogeneous versions of these equations have a solution

$$
R_{N} \sim \Theta \mathrm{e}^{-\chi / \epsilon}, \quad S_{N} \sim Q \mathrm{e}^{-\chi / \epsilon},
$$

corresponding to gravity waves on the free surface. Following Dingle (1973) we expect there to be Stokes lines whenever successive terms in the expansions of $\theta$ and $q$ have the same phase, i.e. when $\chi$ is real and positive. We will show that a multiple of (4.3) is switched on across these Stokes lines. We define the Stokes multiplier $A$ by setting

$$
S_{N}=A Q \mathrm{e}^{-\chi / \epsilon} .
$$

Then, using (4.1) in (4.2) and noting (3.11) and (3.14), gives

$$
\epsilon q_{0}^{3} \frac{\mathrm{d} A}{\mathrm{~d} w} Q \mathrm{e}^{-\chi / \epsilon} \sim \mathrm{i} \epsilon^{N} q_{N},
$$

as $\epsilon \rightarrow 0$ and $N \rightarrow \infty$. Changing variable to write $A$ as a function of $\chi$ instead of $w$, and using the expression (3.17) for $q_{N}$ gives

$$
\frac{\mathrm{d} A}{\mathrm{~d} \chi}=\frac{\epsilon^{N-1} \mathrm{e}^{\chi / \epsilon} \Gamma(N+\gamma)}{\chi^{N+\gamma}} .
$$

Now the right-hand side of (4.4) is minimal for $N \sim|\chi| / \epsilon$, corresponding to the optimal truncation point. Hence we let $N=|\chi| / \epsilon+\rho$, where $\rho$ is bounded as $\epsilon \rightarrow 0$. Then, since $N$ is a function of $|\chi|$ only, following Chapman et al. (1998) we write $\chi=r \mathrm{e}^{\mathrm{i} \vartheta}$ and write

$$
\frac{\mathrm{d}}{\mathrm{d} \chi}=-\frac{\mathrm{ie}^{-\mathrm{i} \vartheta}}{r} \frac{\mathrm{d}}{\mathrm{d} \vartheta} \text {. }
$$

Then, as $\epsilon \rightarrow 0$, using Stirling's approximation of the $\Gamma$-function,

$$
\begin{aligned}
\frac{\mathrm{d} A}{\mathrm{~d} \vartheta} & \sim \frac{\mathrm{i} \sqrt{2 \pi} r \exp (\mathrm{i} \vartheta) \epsilon^{N+\gamma} \exp (r \exp (\mathrm{i} \vartheta) / \epsilon) \exp (-N-\gamma)(N+\gamma)^{N+\gamma-1 / 2}}{\epsilon^{1+\gamma} r^{N+\gamma} \exp (\mathrm{i}(N+\gamma) \vartheta)} \\
& \sim \frac{\mathrm{i} \sqrt{2 \pi} r^{1 / 2} \exp (i \vartheta) \exp (r \exp (i \vartheta) / \epsilon) \exp (-r / \epsilon-\rho-\gamma)}{\epsilon^{1 / 2+\gamma} \exp (\mathrm{i}(r / \epsilon+\rho+\gamma) \vartheta)}\left(1+\frac{\epsilon(\rho+\gamma)}{r}\right)^{r / \epsilon+\rho+\gamma} \\
& \sim \frac{\mathrm{i} \sqrt{2 \pi} r^{1 / 2} \exp (i \vartheta) \exp (r \exp (i \vartheta) / \epsilon) \exp (-r / \epsilon)}{\epsilon^{1 / 2+\gamma} \exp (\mathrm{i} \vartheta(r / \epsilon+\rho+\gamma))}
\end{aligned}
$$

Now, the right-hand side is exponentially small except near $\vartheta=0$, which is the Stokes line. Thus there is a rapid variation in $A$ at this point, corresponding to the switching on of our subdominant exponential. To examine this transition we introduce the local 
scaling $\vartheta=\delta \bar{\vartheta}$, giving

$$
\frac{\mathrm{d} A}{\mathrm{~d} \bar{\vartheta}} \sim \frac{\mathrm{i} \sqrt{2 \pi} r^{1 / 2} \delta \exp \left(-r \delta^{2} \bar{\vartheta}^{2} /(2 \epsilon)\right)}{\epsilon^{1 / 2+\gamma}} .
$$

We see that the correct scaling is $\delta=\epsilon^{1 / 2}$, giving

$$
\frac{\mathrm{d} A}{\mathrm{~d} \bar{\vartheta}} \sim \frac{\mathrm{i} \sqrt{2 \pi} r^{1 / 2} \exp \left(-r \bar{\vartheta}^{2} / 2\right)}{\epsilon^{\gamma}} .
$$

Hence

$$
A=A_{0}+\frac{\sqrt{2 \pi} \mathrm{i}}{\epsilon^{\gamma}} \int_{-\infty}^{\vartheta \sqrt{r}} \exp \left(-t^{2} / 2\right) \mathrm{d} t .
$$

Matching with the outer solution away from the Stokes line we find there is a jump in $A$ given by

$$
A(\vartheta=0-)-A(\vartheta=0+)=\frac{2 \pi \mathrm{i}}{\epsilon^{\gamma}} .
$$

Thus, by optimally truncating the expansion in powers of $\epsilon$ and examining the equation for the remainder, we have been able to observe directly subdominant exponentials being switched on across Stokes lines.

To summarize, the Stokes lines are the lines on which $\chi$ is real and positive, and across these Stokes lines an exponentially small correction term to the optimally truncated algebraic expansion of $q$ is switched on, which is

$$
\frac{2 \pi \mathrm{i}}{\epsilon^{\gamma}} Q \exp (-\chi / \epsilon)
$$

Since $R_{n} \sim-\mathrm{i} S_{n} / q_{0}$ by (4.1) this means that, using (3.17), the corresponding exponentially small correction term in $\theta$ is

$$
\frac{2 \pi \mathrm{i}}{\epsilon^{\gamma}} \Theta \exp (-\chi / \epsilon)
$$

For each singularity in the upper half-plane, there will be a corresponding singularity in the lower half-plane for which the exponentially small correction terms are the complex conjugates of (4.6), (4.7). Thus, for real $\xi$ (i.e. on the real free surface), the correction term is equal to twice the real part of (4.6), (4.7). Since $\chi$ is complex, these exponentials are oscillatory, i.e. they correspond to gravity waves on the free surface.

Let us now examine the implications of the analysis of the preceeding two sections on a concrete example, namely the flow over a step.

\section{Example: flow over a step}

As a concrete example to illustrate the ideas we have introduced, we consider the flow over a step as shown in figure 1 . This problem was previously analysed by King \& Bloor (1987), who found the height of the waves on the free surface analytically in the limit of small step height for arbitrary Froude number. Our analysis is applicable in the complementary regime of arbitrary step height but small Froude number, and it is interesting to compare the two approximations.

If the flow at $-\infty$ is uniform of speed $U$, and the height of the channel at $-\infty$ is $h$, we non-dimensionalize velocity with $U$ and length with $h / \pi$, so that the streamfunction varies from zero on the free surface to $-\pi$ on the channel bottom. Then $\epsilon=\pi U^{2} / g h$. 
(a)

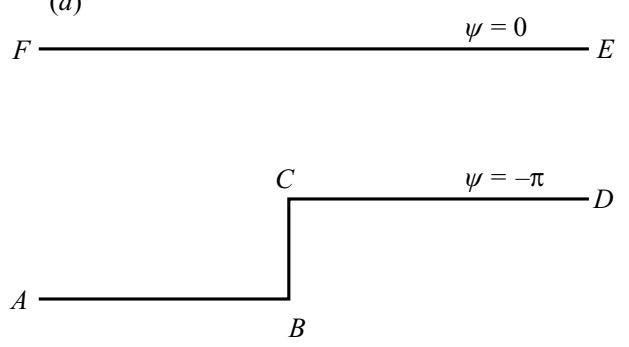

(b)

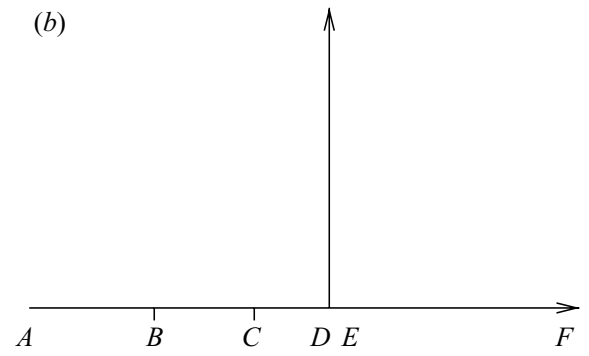

FIGURE 1. Flow over a step. (a) z-plane; (b) $\zeta$-plane.

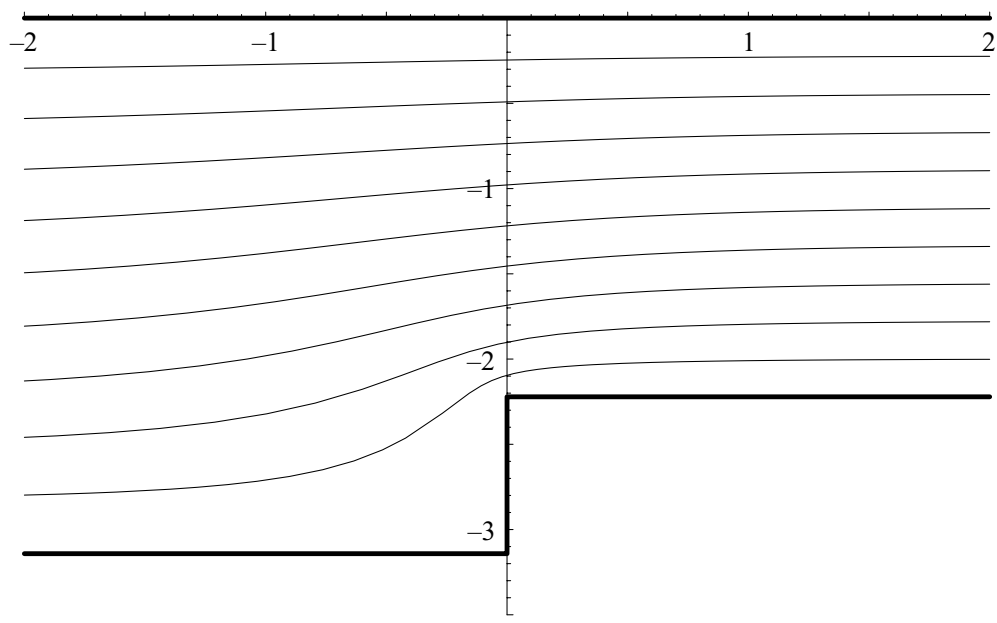

FIGURE 2. Streamlines for flow over a step at zero Froude number $(\epsilon=0)$, with $a=1, b=2$.

The only part of the analysis in $\S \S 3$ and 4 which depends on the particular geometry under consideration is the map $w \rightarrow \zeta$ from the potential plane to the upper-half- $\zeta-$ plane. For the case of flow over a step, this map is $\zeta=\mathrm{e}^{-w}$. If we write the equations in terms of $\zeta$ we have

$$
\begin{aligned}
\epsilon q^{2} \zeta \frac{\mathrm{d} q}{\mathrm{~d} \zeta} & =\sin \theta \\
\log q & =\frac{1}{2} \log \left(\frac{\zeta+b}{\zeta+a}\right)-\frac{1}{\pi} f_{0}^{\infty} \frac{\theta\left(\zeta^{\prime}\right) \mathrm{d} \zeta^{\prime}}{\zeta^{\prime}-\zeta},
\end{aligned}
$$

where $0<a<b$ and the free boundary corresponds to $0<\zeta<\infty ;-a$ and $-b$ are the images of the corners of the step $C$ and $B$ in the $\zeta$-plane. The leading-order solution is

$$
\theta_{0}=0, \quad q_{0}=\left(\frac{\zeta+b}{\zeta+a}\right)^{1 / 2}
$$

so that the non-dimensional step height is $\pi\left(1-(a / b)^{1 / 2}\right)$, to leading order. The corresponding streamlines for the case $a=1, b=2$ are shown in figure 2 .

The singularities of $q_{0}$ lie at $\zeta=-b$ and $\zeta=-a$, i.e. at the corners of the step. Since the singularity at $\zeta=-b$ corresponds to an interior angle less than $2 \pi / 3$, it does not 


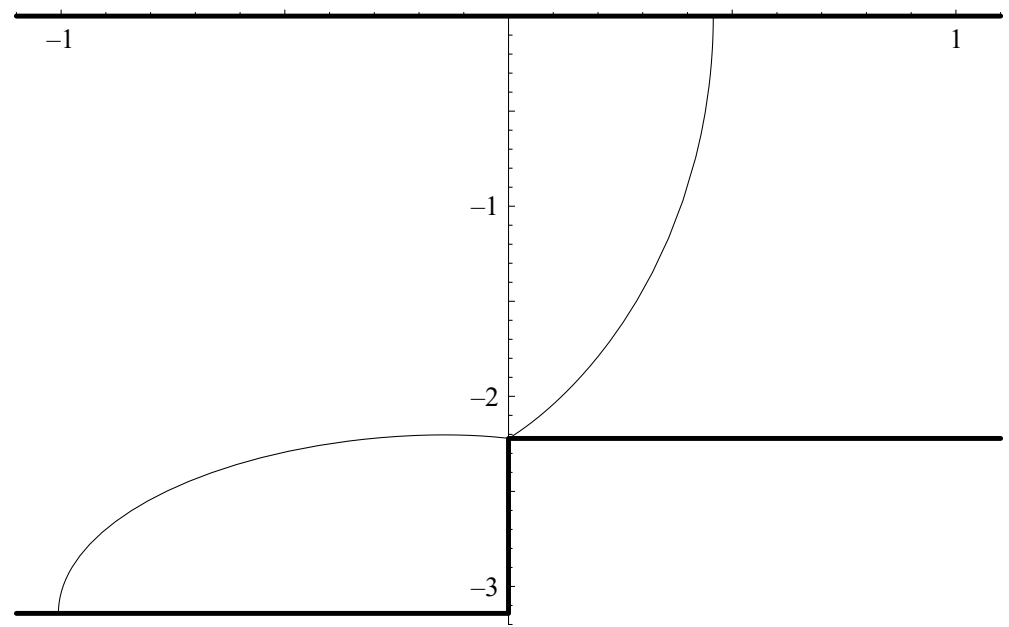

FiguRE 3. Stokes lines for flow over a step, with $a=1, b=2$.

generate any Stokes lines. Thus, the important singularity is that at $\zeta=-a$, giving

$$
\chi=-\mathrm{i} \int_{w_{0}}^{w} \frac{\mathrm{d} w}{q_{0}^{3}}=\mathrm{i} \int_{-a}^{\zeta}\left(\frac{\zeta+a}{\zeta+b}\right)^{3 / 2} \frac{\mathrm{d} \zeta}{\zeta} .
$$

The Stokes lines, corresponding to $\chi$ real and positive, are shown in figure 3 for the case $a=1, b=2$, for which $\chi$ can be evaluated explicitly as

$$
\chi=-i \sqrt{\frac{1+\zeta}{2+\zeta}}+2 i \sinh ^{-1} \sqrt{1+\zeta}-\frac{i}{\sqrt{2}} \tanh ^{-1} \sqrt{\frac{2(1+\zeta)}{2+\zeta}} .
$$

There is one Stokes line which meets the free boundary, across which exponentially small gravity waves will be turned on. To determine the amplitude of the gravity waves switched on across this Stokes line we must evaluate $\gamma, c$ and $C$ so that we can determine $\Lambda$. As $\zeta \rightarrow-a$,

$$
q_{0} \sim \frac{(b-a)^{1 / 2}}{(\zeta+a)^{1 / 2}}, \quad w \sim-\log a-\mathrm{i} \pi+\frac{\zeta+a}{a},
$$

so that $w_{0}=-\log a-\mathrm{i} \pi$ and

$$
q_{0} \sim \frac{(b-a)^{1 / 2}}{a^{1 / 2}\left(w-w_{0}\right)^{1 / 2}}
$$

Hence,

and, from (3.22),

$$
\alpha=-\frac{1}{2}, \quad c=\frac{(b-a)^{1 / 2}}{a^{1 / 2}},
$$

$$
\gamma=\frac{6}{5}
$$

To determine $C$ in (3.19) we must evaluate $q_{1}$. We have

$$
\begin{aligned}
q_{0}^{2} \zeta \frac{\mathrm{d} q_{0}}{\mathrm{~d} \zeta} & =\theta_{1}, \\
\frac{q_{1}}{q_{0}} & =-\frac{1}{\pi} f_{0}^{\infty} \frac{\theta_{1}\left(\zeta^{\prime}\right) \mathrm{d} \zeta^{\prime}}{\zeta^{\prime}-\zeta},
\end{aligned}
$$


so that

$$
C=q_{0}^{3}\left(\zeta^{*}\right) \exp \left(-3 \mathrm{i} \int_{\zeta^{*}}^{-a} \frac{1}{\pi q_{0}^{3}} \int_{0}^{\infty} \frac{\theta_{1}\left(\zeta^{\prime}\right) \mathrm{d} \zeta^{\prime}}{\zeta^{\prime}-\zeta} \frac{\mathrm{d} \zeta}{\zeta}\right)
$$

where $w^{*}=-\log \zeta^{*}$. For the amplitude of gravity waves, it is the modulus of $C$ which is important. Noting that for real $\zeta$

$$
\operatorname{Im}\left(\frac{1}{\pi} \int_{0}^{\infty} \frac{\theta_{1}\left(\zeta^{\prime}\right) \mathrm{d} \zeta^{\prime}}{\zeta^{\prime}-\zeta}\right)= \begin{cases}0 & \zeta<0 \\ \theta_{1} & \zeta>0\end{cases}
$$

we find

$$
\begin{aligned}
\operatorname{Re}\left(-3 \mathrm{i} \int_{\zeta^{*}}^{-a} \frac{1}{\zeta q_{0}^{3} \pi} \int_{0}^{\infty} \frac{\theta_{1}\left(\zeta^{\prime}\right) \mathrm{d} \zeta^{\prime}}{\zeta^{\prime}-\zeta} \mathrm{d} \zeta\right)=3 \int_{\zeta^{*}}^{0} \frac{1}{q_{0}} \frac{\mathrm{d} q_{0}}{\mathrm{~d} \zeta} d \zeta+\frac{3}{q_{0}(0)^{3}} \int_{0}^{\infty} \frac{\theta_{1}\left(\zeta^{\prime}\right) \mathrm{d} \zeta^{\prime}}{\zeta^{\prime}} \\
=3 \log q_{0}(0)-3 \log q_{0}\left(\zeta^{*}\right)+\frac{3}{q_{0}(0)^{3}} \int_{0}^{\infty} \frac{\theta_{1}\left(\zeta^{\prime}\right) \mathrm{d} \zeta^{\prime}}{\zeta^{\prime}}
\end{aligned}
$$

where the first integral is from $\zeta>0$ and the second is due to the pole at the origin. Now

$$
\int_{0}^{\infty} \frac{\theta_{1}\left(\zeta^{\prime}\right) \mathrm{d} \zeta^{\prime}}{\zeta^{\prime}}=\int_{0}^{\infty} q_{0}^{2} \frac{\mathrm{d} q_{0}}{\mathrm{~d} \zeta} \mathrm{d} \zeta=\left[\frac{q_{0}^{3}}{3}\right]_{0}^{\infty}=\frac{1}{3}\left(1-q_{0}(0)^{3}\right)
$$

Thus,

$$
\operatorname{Re}\left(-3 \mathrm{i} \int_{\zeta^{*}}^{-a} \frac{1}{\zeta q_{0}^{3} \pi} \int_{0}^{\infty} \frac{\theta_{1}\left(\zeta^{\prime}\right) \mathrm{d} \zeta^{\prime}}{\zeta^{\prime}-\zeta} \mathrm{d} \zeta\right)=3 \log q_{0}(0)-3 \log q_{0}\left(\zeta^{*}\right)+\frac{1}{q_{0}^{3}(0)}-1
$$

Hence, combining (5.5) and (5.7), and using (5.3), we have

$$
|C|=\frac{b^{3 / 2}}{a^{3 / 2}} \exp \left(\frac{a^{3 / 2}}{b^{3 / 2}}-1\right)
$$

We are now in a position to determine $|\Lambda|$. Iterating (3.29) to large values of $n$, we find

$$
\lim _{n \rightarrow \infty} \frac{\phi_{n}}{\Gamma(n+\gamma)} \approx 0.389364
$$

Hence, from (3.32),

$$
\Lambda C \approx \frac{0.389364 \mathrm{e}^{3 i \pi / 5}}{2(5 / 2)^{6 / 5}}\left(\frac{b}{a}-1\right)^{6 / 5} \approx 0.065 \mathrm{e}^{3 i \pi / 5}\left(\frac{b}{a}-1\right)^{6 / 5},
$$

so that

$$
|\Lambda| \approx 0.065\left(\frac{b}{a}-1\right)^{6 / 5}\left(\frac{a}{b}\right)^{3 / 2} \exp \left(1-\frac{a^{3 / 2}}{b^{3 / 2}}\right) .
$$

From (4.7) and (3.16), the exponentially small correction term to the truncated expansion of $\theta$ which is switched on across the Stokes line is

$$
\begin{aligned}
\theta_{\exp } & =\frac{2 \pi}{\epsilon^{\gamma}} \frac{\Lambda}{q_{0}^{3}} \exp \left(-3 \mathrm{i} \int_{w^{*}}^{w} \frac{q_{1}}{q_{0}^{4}} \mathrm{~d} w\right) \exp (-\chi / \epsilon)+\text { complex conjugate } \\
& =\frac{4 \pi}{\epsilon^{6 / 5}} \frac{|\Lambda|}{q_{0}^{3}} \exp (-\operatorname{Re}(\chi) / \epsilon) \cos \left(-3 \int_{w^{*}}^{w} \frac{q_{1}}{q_{0}^{4}} \mathrm{~d} w-\frac{\operatorname{Im}(\chi)}{\epsilon}+\arg (\Lambda)\right) .
\end{aligned}
$$


The real part of $\chi$ comes from the pole at the origin of $\zeta$, giving $\operatorname{Re}(\chi)=a^{3 / 2} b^{-3 / 2} \pi$. Now, as $x \rightarrow \infty$, we have

$$
\zeta \rightarrow 0, \quad q_{0} \rightarrow\left(\frac{b}{a}\right)^{1 / 2}, \quad w \sim x\left(\frac{b}{a}\right)^{1 / 2}, \quad \chi \sim-\mathrm{i} w\left(\frac{a}{b}\right)^{3 / 2} \sim-\mathrm{i} x\left(\frac{a}{b}\right) .
$$

Thus, as $x \rightarrow \infty$, using (5.3) and (5.10) in (5.11),

$$
\begin{aligned}
\theta_{\exp } \sim \frac{0.82}{\epsilon^{6 / 5}}\left(\frac{b}{a}-1\right)^{6 / 5}\left(\frac{a}{b}\right)^{3} & \exp \left(1-\frac{a^{3 / 2}}{b^{3 / 2}}-\frac{\pi a^{3 / 2}}{\epsilon b^{3 / 2}}\right) \\
& \times \cos \left(-3 \int_{w^{*}}^{w} \frac{q_{1}}{q_{0}^{4}} \mathrm{~d} w+\frac{x a}{\epsilon b}+\text { constant }\right) .
\end{aligned}
$$

Since the equation of the free surface is

$$
\frac{\mathrm{d} y}{\mathrm{~d} x}=\tan \theta \sim \theta
$$

for small $\theta$, this correction term to $\theta$ gives a corresponding exponentially small oscillation in the height of the free surface

$$
\begin{aligned}
y_{\exp } \sim \frac{0.82}{\epsilon^{1 / 5}}\left(\frac{b}{a}-1\right)^{6 / 5}\left(\frac{a}{b}\right)^{2} \exp ( & \left.1-\frac{a^{3 / 2}}{b^{3 / 2}}-\frac{\pi a^{3 / 2}}{\epsilon b^{3 / 2}}\right) \\
& \times \sin \left(-3 \int_{w^{*}}^{w} \frac{q_{1}}{q_{0}^{4}} \mathrm{~d} w+\frac{x a}{\epsilon b}+\text { constant }\right) .
\end{aligned}
$$

Thus, the amplitude of the gravity waves on the free surface at infinity is

$$
H \sim \frac{0.82}{\epsilon^{1 / 5}}\left(\frac{b}{a}-1\right)^{6 / 5}\left(\frac{a}{b}\right)^{2} \exp \left(1-\frac{a^{3 / 2}}{b^{3 / 2}}-\frac{\pi a^{3 / 2}}{\epsilon b^{3 / 2}}\right) .
$$

Although (5.13) is asymptotically correct for small $\epsilon$, it is not as good an approximation as (5.12). When we integrate $\theta$ to obtain $y$ we effectively divide by the wavenumber of the waves. Rather than using the asymptotic value for the wavenumber of $a / \epsilon b$, which may not be so accurate when $\epsilon b$ is not too small (as in our examples), we can simply use the exact value for the wavenumber of small-amplitude waves at infinity, given by $1 / \epsilon q_{\infty}^{2}$ where

$$
\frac{a^{1 / 2}}{b^{1 / 2}}-\frac{1}{q_{\infty}}=\frac{\epsilon}{2 \pi}\left(q_{\infty}^{2}-1\right)
$$

we do this when we compare the asymptotic solution to a full numerical solution in $\S 6$. Note that for sufficiently large $b,(5.14)$ has no solution, indicating that for such steps, the waves at infinity are not small, but of order one amplitude.

Before we compare (5.12) to a numerical simulation, we first compare our result for an arbitrary step height at small Froude number with that of a small step at arbitrary Froude number, a result due to King \& Bloor (1987). In the limit of small step height, the images in the $\zeta$-plane of the top and bottom of the step are close together. Thus, $b=a(1+\delta), \delta \ll 1$. In this case, the problem can be linearized in $\delta$ at arbitrary Froude number, and the amplitude of the gravity waves generated is given by King \& Bloor (1987) as (in our scaling)

$$
H \sim \frac{\pi \delta \epsilon}{\cosh k\left(\epsilon-\operatorname{sech}^{2} k\right)},
$$


where $k$ is the positive root of

$$
\epsilon k=\tanh k .
$$

As $\epsilon \rightarrow 0, k \sim 1 / \epsilon$ and the amplitude of the gravity waves is

$$
H \sim 2 \pi \delta \mathrm{e}^{-\pi / \epsilon} .
$$

Now, if we write $b=a(1+\delta)$ in (5.13) and take the limit as $\delta \rightarrow 0$, we find that the amplitude of gravity waves is approximately

$$
H \sim 0.82 \epsilon^{-1 / 5} \delta^{6 / 5} \mathrm{e}^{-\pi / \epsilon} .
$$

This clearly does not agree with (5.16), and we can see why by looking at (5.13). It is clear from looking at the exponential that there is a distinguished limit when $\delta=O(\epsilon)$; the limits $\delta \rightarrow 0$ and $\epsilon \rightarrow 0$ do not commute. In Appendix A, we consider the limit in which $\delta \ll \epsilon$, so that we first linearize the equations in $\delta$, and then perform our beyond-all-orders analysis. In this limit, we retrieve the result (5.16) of King \& Bloor (1987). In Appendix B we examine the distinguished limit $\delta=O(\epsilon)$.

Finally, we comment on the corresponding results for a step down rather than a step up. In fact, our results do not depend on the direction of the flow, and could equally well be applied to flow from right to left down the same step. The Stokes line analysis tells us the jump in the amplitude of the waves as we cross the Stokes line; it does not say which side the waves are present. To determine the position of the waves, we applied the radiation condition so that there were no waves arriving from infinity, that is, the waves are present downstream.

Thus, if we reverse the flow, we would find waves present at minus infinity rather than plus infinity. Expression (5.11) still holds, but now is evaluated as $x \rightarrow-\infty$, where

$$
\zeta \rightarrow \infty, \quad q_{0} \rightarrow 1, \quad w \sim x, \quad \chi \sim-\mathrm{i} w \sim-\mathrm{i} x .
$$

Thus, as $x \rightarrow-\infty$,

$$
\begin{aligned}
\theta_{\exp } \sim \frac{0.82}{\epsilon^{6 / 5}}\left(\frac{b}{a}-1\right)^{6 / 5}\left(\frac{a}{b}\right)^{3 / 2} \exp ( & \left.1-\frac{a^{3 / 2}}{b^{3 / 2}}-\frac{\pi a^{3 / 2}}{\epsilon b^{3 / 2}}\right) \\
& \times \cos \left(-3 \int_{w^{*}}^{w} \frac{q_{1}}{q_{0}^{4}} \mathrm{~d} w+x+\text { constant }\right),
\end{aligned}
$$

giving the amplitude of the gravity waves on the free surface at minus infinity as

$$
H \sim \frac{0.82}{\epsilon^{1 / 5}}\left(\frac{b}{a}-1\right)^{6 / 5}\left(\frac{a}{b}\right)^{3 / 2} \exp \left(1-\frac{a^{3 / 2}}{b^{3 / 2}}-\frac{\pi a^{3 / 2}}{\epsilon b^{3 / 2}}\right) .
$$

Alternatively, we can observe that the situation considered previously corresponds to a step down rather than a step up if $0<b<a$ rather than $0<a<b$. The important singularity is still $\zeta=-a$, and the same methodology can be employed. Now, however, care must be taken when evaluating some of the integrals. For example, in evaluating the real part of $\chi$ as $\zeta \rightarrow \infty$ from (5.4) we do not now want to integrate along the real axis in the region $-a<\zeta<-b$ where the argument of the root is negative. Instead we integrate to minus infinity, and then round in a large cirular arc to arrive at plus infinity. Thus, the real part of $\chi$ arises from the pole at infinity in this case, rather than the pole at zero. 


\section{Numerical results}

In this section we describe a boundary-integral-equation method to compute fully nonlinear solutions for the flow over a step. The numerical procedure is similar to that used by King \& Bloor (1987), Tuck \& Vanden-Broeck (1998) and Chapman \& Vanden-Broeck (2002).

We truncate the domain to $\left[X_{-}, X_{+}\right]$, and introduce the equally spaced points in $\phi$

$$
\phi_{I}=X_{-}+\left(\frac{I-1}{N-1}\right)\left(X_{+}-X_{-}\right) \quad(I=1, \ldots, N)
$$

and the corresponding unknowns

$$
\theta_{I}=\theta\left[\zeta\left(\phi_{I}\right)\right] \quad(I=1, \ldots, N)
$$

where $\zeta=\mathrm{e}^{-\phi}$.

Writing $\tau=\log q$, we evaluate the values $\tau_{I}^{M}$ of $\tau[\zeta(\phi)]$ at the midpoints

$$
\phi_{I}^{M}=\frac{\phi_{I}+\phi_{I+1}}{2} \quad(I=1, \ldots, N-1),
$$

by applying the trapezoidal rule to the integral in (5.2) with a summation over the mesh points $\phi_{I}$. The use of equally spaced points and of midpoints enables us to neglect the singularity of the Cauchy principal value without losing accuracy.

The values of $\theta$ at the midpoints are evaluated in terms of the unknowns (6.2) by interpolation formulae. Next, the values of $y$ at the midpoints are obtained by integrating the identity

$$
\frac{\partial y}{\partial \phi}=\mathrm{e}^{-\tau} \sin \theta
$$

by the trapezoidal rule with the condition $y\left(\phi_{1}\right)=0$. Next we satisfy Bernoulli's equation

$$
\epsilon\left(\frac{q^{2}}{2}-\frac{1}{2}\right)+y=0
$$

at the midpoints $\phi_{I}^{M}$. For given values of $a, b$ and $\epsilon$, this gives a system of $N-1$ nonlinear algebraic equations for the $N$ unknowns, (6.2). The last equation imposes the radiation condition by requiring $\theta_{1}=0$. This forces the free surface to be flat and waveless as $x \rightarrow-\infty$. The system of $N$ equations with $N$ unknowns is solved by the Newton method.

There are two sources of error in the computations, namely the values $X_{-}$and $X_{+}$at which the domain is truncated and the number of mesh points $N$. For given values of $X_{-}$and $X_{+}$, we run the program for increasing values of $N$ until the results are independent of $N$ within graphical accuracy. We then repeat the procedure for larger values of $X_{-}$and $X_{+}$until the results are also independent of $X_{-}$and $X_{+}$. Most of the results below could be calculated with $N=1300, X_{-}=-12$ and $X_{+}=4$. Note that, although the truncation error is $K \delta \phi^{2}$, with $\delta \phi^{2}$ about $10^{-4}$, we are able to calculate accurately the amplitude of gravity waves which are much smaller than $10^{-4}$. This is because for small amplitude waves, the constant $K$ is also very small.

The results of the numerical simulations are shown in figures 4-8. In figure 4, we show an example of the profile of the free surface, illustrating the waves generated by the step. In figure 5, we show the logarithm of the amplitude of the wavetrain at infinity as a function of $b$, for $\epsilon=0.04 \pi$ (corresponding to a Froude number of 0.35 ). The asymptotic results for $b-a=O(1)$ (equation (5.12)) and $b-a=O(\epsilon)$ (equation (B 16)) are shown, along with a uniform approximation generated by adding 


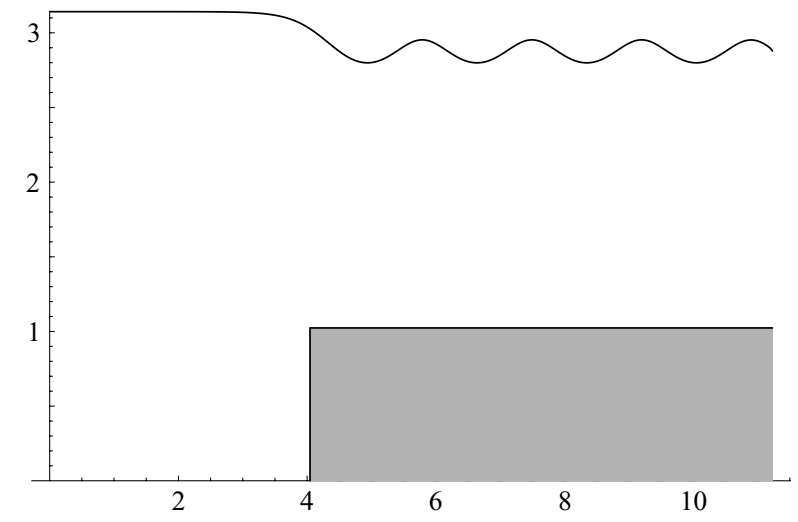

FIGURE 4. The numerically calculated free surface for $a=1, b=2.2$ and $\epsilon=0.3$, corresponding to a Froude number of 0.548 .

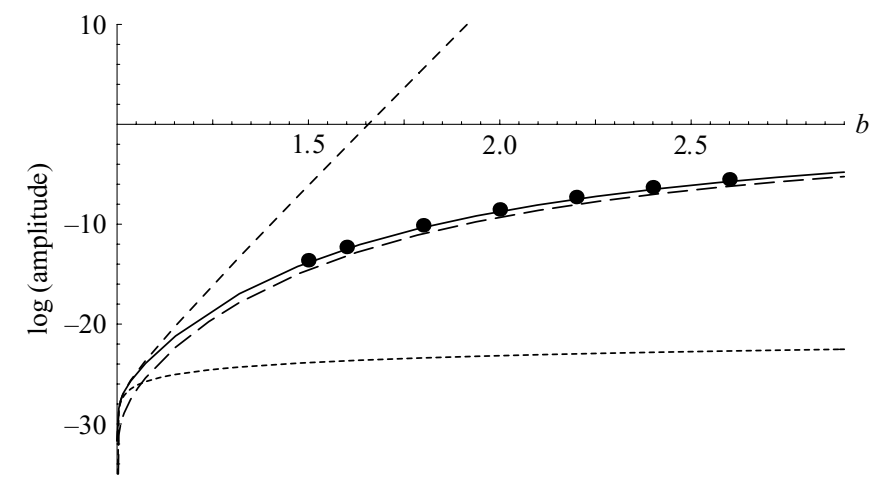

FIGURE 5. The natural logarithm of the amplitude of the wavetrain at infinity as a function of $b$, for $a=1$ and $\epsilon=0.04 \pi$, corresponding to a Froude number of 0.35 . The dotted curve is the linearized theory of King \& Bloor with $b-a \ll \epsilon$ (equation (5.15)), the long dashed line is the outer approximation with $b-a=O(1)$ (equation (5.12)), and the dashed curve is the asymptotic approximation when $b-a=O(\epsilon)$ (equation (B 16)). The solid curve is the uniform approximation generated from these last two. The points are the numerical simulation.

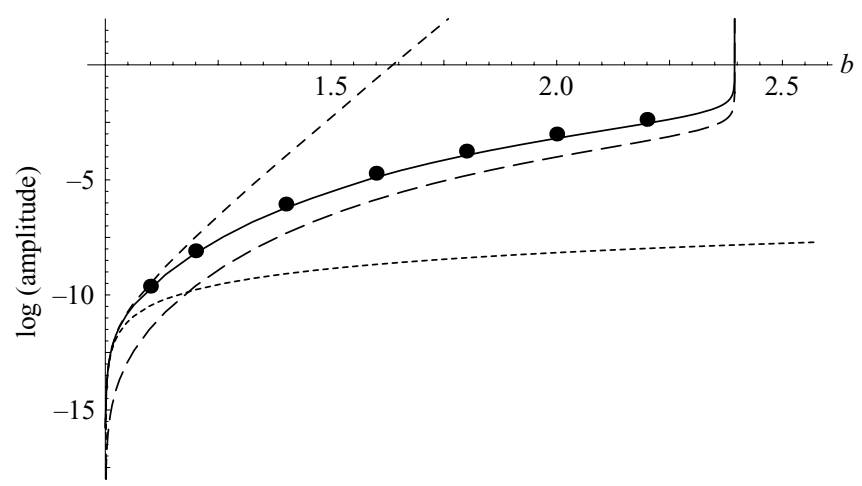

Figure 6. As for figure 5, but $\epsilon=0.1 \pi$ and the Froude number is 0.56 . 


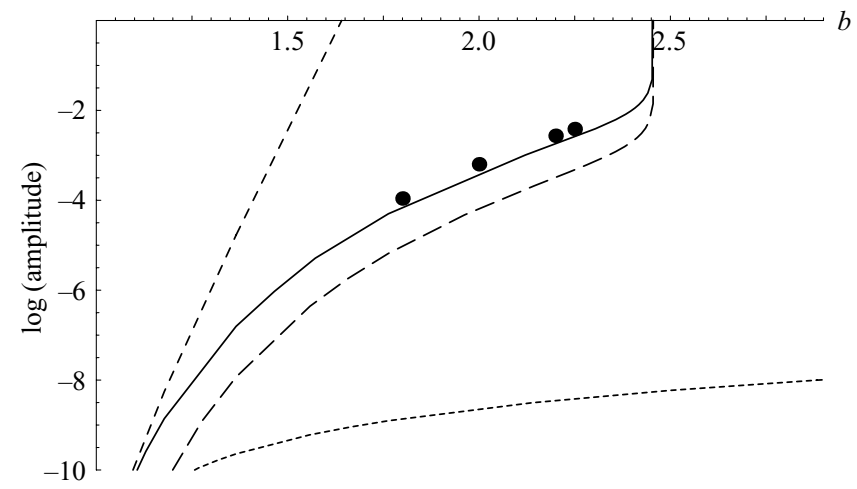

FIGURE 7. As for figure 5, but $\epsilon=0.3$ and the Froude number is 0.548 .

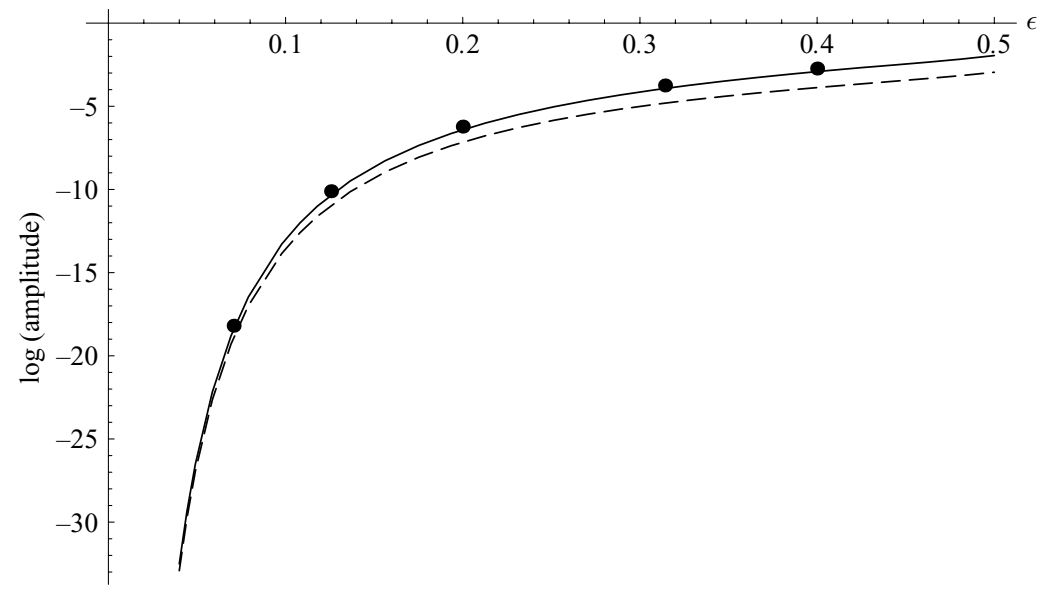

FIGURE 8 . The natural logarithm of the amplitude of the wave train at infinity as a function of $\epsilon$, for $a=1$ and $b=1.8$. The long dashed line is the outer approximation with $b-a=O(1)$ (equation (5.12)), and the solid curve is the uniform approximation. The points are the numerical simulation.

the logarithms of these two results and subtracting the overlap. Also shown is the linearized small-step result of King \& Bloor (1987) (equation (5.15)). The points are the numerical simulations. We see that the outer approximation is very good, and the uniform approximation even better, whereas the small-step approximation is good only for $b$ very close to $a$, when the waves are extremely small.

In figure 6 , we show the results for $\epsilon=0.1 \pi$, corresponding to a Froude number of 0.56. In this case, because the Froude number is not that small, we see that there is a large difference between the outer approximation with $b-a=O(1)$ and the uniform approximation. However, the uniform approximation is still close to the numerical results. Even for this relatively large Froude number, the small-step approximation is good only for $b$ very close to $a$.

In figure 7, we show the results for $\epsilon=0.3$, corresponding to a Froude number of 0.548 , the same as that used in figure 4 . Even though the waves are not small, the asymptotic approximation is still good.

Finally, in figure 8, we show the logarithm of the amplitude of the wavetrain at infinity as a function of $\epsilon$, for $a=1$ and $b=1.8$. Here we can see that as $\epsilon$ is increased, 
the error in the outer approximation with $b-a=O(1)$ gradually increases. However, the uniform approximation is still showing no signs of divergence, even at $\epsilon=0.4$, corresponding to a Froude number of 0.63 .

Note that the logarithms in figures 5-8 are all natural logarithms, so that while the amplitude of the waves is exponentially small in $\epsilon$, and is numerically very small for small Froude number, the asymptotic results provide a good approximation even for Froude numbers in the range 0.5-0.7, with corresponding wave amplitudes around 0.08 . As illustrated in figure 4 , such amplitudes are not particularly small, so that our results may have practical as well as theoretical significance.

\section{Conclusion}

We have examined the problem of irrotational inviscid incompressible free-surface flows in the limit of small Froude number. We find that singularities in the complexification of the surface velocity result in a divergent asymptotic expansion in powers of the Froude number. Such singularities correspond to stagnation points or corners in submerged objects or on rough beds. The divergence of the asymptotic expansion is associated with Stokes phenomenon. By optimally truncating the expansion, we are able to observe exponentially small gravity waves generated across Stokes lines.

As an example of the application of our theory, we considered the flow over a step previously considered by King \& Bloor (1987). We found that there were, in fact, three possible parameter regimes, depending on whether the dimensionless step height was small, of the same order, or large compared to the square of the Froude number.

Although the amplitude of the waves we predict is exponentially small in the Froude number and therefore numerically very small for small Froude numbers, numerical simulations of the full nonlinear problem show that our asymptotic results are accurate even for quite large Froude numbers, for which the amplitude of the waves is around 0.08 , and is significant. This is in contrast to the linearized theory of small step height, which is accurate only for very small steps.

\section{Appendix A. Small step height}

In this section, we consider the limit in which $\delta \ll \epsilon$, so that we first linearize the equations in $\delta$, and then perform our beyond-all-orders analysis. In this limit, we should retrieve the result (5.16) of King \& Bloor (1987). In the following section, we examine the distinguished limit in which $\delta=O(\epsilon)$.

With $b=a(1+\delta), \delta \ll 1$ we expand

$$
\begin{aligned}
& q=1+\delta q^{\prime}+\cdots, \\
& \theta=\delta \theta^{\prime}+\cdots,
\end{aligned}
$$

to give, to leading order in $\delta$ (dropping the primes)

$$
\begin{aligned}
\epsilon \zeta \frac{\mathrm{d} q}{\mathrm{~d} \zeta} & =\theta, \\
q & =\frac{1}{2(\zeta+a)}-\frac{1}{\pi} f_{0}^{\infty} \frac{\theta\left(\zeta^{\prime}\right) \mathrm{d} \zeta^{\prime}}{\zeta^{\prime}-\zeta} \\
& =\frac{1}{2(\zeta+a)}+\mathrm{i} \theta-\frac{1}{\pi} \int_{0}^{\infty} \frac{\theta\left(\zeta^{\prime}\right) \mathrm{d} \zeta^{\prime}}{\zeta^{\prime}-\zeta},
\end{aligned}
$$


where we indent under the pole in the integral in (A 2) so that we can analytically continue $\zeta$ to the upper half-plane. We now very briefly repeat the analysis of $\S 3$ on the new equations (A 1)-(A 2). Expanding

$$
\theta=\sum_{n=0}^{\infty} \epsilon^{n} \theta_{n}, \quad q=\sum_{n=0}^{\infty} \epsilon^{n} q_{n},
$$

the leading-order solution is

$$
\theta_{0}=0, \quad q_{0}=\frac{1}{2(\zeta+a)}
$$

while at $O\left(\epsilon^{n}\right)$

$$
\begin{aligned}
\zeta \frac{\mathrm{d} q_{n-1}}{\mathrm{~d} \zeta} & =\theta_{n}, \\
q_{n} & =\mathrm{i} \theta_{n}-\frac{1}{\pi} \int_{0}^{\infty} \frac{\theta_{n}\left(\zeta^{\prime}\right) \mathrm{d} \zeta^{\prime}}{\zeta^{\prime}-\zeta} .
\end{aligned}
$$

With the ansatz (3.8), the final integral in (A 5) is exponentially subdominant, so that (A 5) gives

$$
q_{n} \sim \mathrm{i} \theta_{n} \quad \text { as } n \rightarrow \infty .
$$

Then (A 4) gives, to leading order in $n$ as $n \rightarrow \infty$,

$$
\zeta \frac{\mathrm{d} \chi}{\mathrm{d} \zeta}=\mathrm{i}
$$

so that

$$
\chi=\mathrm{i} \log (-\zeta / a)
$$

where we have used the fact that $\chi(-a)=0$. At the next order in $n$, we find

$$
\frac{\mathrm{d} Q}{\mathrm{~d} \zeta}=0
$$

so that $Q=$ constant $=\Lambda$, say. Now, as $\zeta \rightarrow-a$,

$$
q_{0} \sim \frac{a}{2(\zeta+a)}, \quad \chi \sim-\frac{\mathrm{i}(\zeta+a)}{a} .
$$

For this to be consistent with (3.8) we require $\gamma=1$. Thus the late terms are given by

$$
\theta_{n} \sim-\mathrm{i} \Lambda \frac{\Gamma(n+1)}{\chi^{n+1}}, \quad q_{n} \sim \Lambda \frac{\Gamma(n+1)}{\chi^{n+1}} .
$$

To determine the constant $\Lambda$ we match with an inner expansion near $\zeta=-a$.

\section{A.1. Inner expansion}

In the vicinity of $\zeta=-a$ we have

$$
\begin{aligned}
& q-\mathrm{i} \theta \sim \frac{a}{2(\zeta+a)} \\
& \epsilon \zeta \frac{\mathrm{d} q}{\mathrm{~d} \zeta}=\theta
\end{aligned}
$$


We define the inner coordinate as $\zeta+a=\mathrm{i} a \epsilon \eta$, and set $q=-\mathrm{i} \bar{q} / 2 \epsilon$. Then, to leading order in $\epsilon$,

$$
\frac{\mathrm{d} \bar{q}}{\mathrm{~d} \eta}+\bar{q}=\frac{1}{\eta}
$$

Hence,

$$
\bar{q}=\mathrm{e}^{-\eta} \int_{-\infty}^{\eta} \frac{\mathrm{e}^{s}}{s} \mathrm{~d} s
$$

As $\eta \rightarrow \infty$

$$
\bar{q} \sim \sum_{n=0}^{\infty} \frac{\Gamma(n+1)}{\eta^{n+1}}
$$

so that

$$
q \sim-\frac{\mathrm{i}}{2 \epsilon} \sum_{n=0}^{\infty} \frac{\Gamma(n+1)}{\eta^{n+1}}
$$

The inner limit of the outer expansion (A 8) is

$$
\begin{aligned}
\epsilon^{n} q_{n} & \sim \Lambda \frac{\Gamma(n+1) \epsilon^{n}}{\chi^{n+1}} \\
& =\Lambda \Gamma(n+1) \epsilon^{n}\left(\frac{a}{-\mathrm{i}(\zeta+a)}\right)^{n+1} \\
& =\frac{\Lambda \Gamma(n+1)}{\epsilon \eta^{n+1}}
\end{aligned}
$$

Matching (A 11) and (A 10) we find

$$
\Lambda=-\frac{\mathrm{i}}{2} \text {. }
$$

A.2. Stokes lines and gravity waves

There are Stokes lines whenever $\chi$ is real and positive, corresponding to $|\zeta|=a$ or $\operatorname{Re}(w)=-\log a$. Thus, there is a Stokes line which cuts the real free boundary at $\phi=-\log a$. Across this Stokes line, a calculation identical to that of $\S 4$ shows that the exponentially small correction term

$$
\begin{aligned}
\theta_{\exp } & =\frac{2 \pi \mathrm{i}}{\epsilon^{\gamma}} \Theta e^{-x / \epsilon}+\text { complex conjugate } \\
& =-\frac{2 \pi}{\epsilon} \mathrm{e}^{-\pi / \epsilon} \sin \left(\frac{\log (\zeta / a)}{\epsilon}\right) \\
& =\frac{2 \pi}{\epsilon} \mathrm{e}^{-\pi / \epsilon} \sin \left(\frac{\phi}{\epsilon}+\frac{\log a}{\epsilon}\right) \\
& =\frac{2 \pi}{\epsilon} \mathrm{e}^{-\pi / \epsilon} \sin \left(\frac{x}{\epsilon}+\frac{\log a}{\epsilon}\right)
\end{aligned}
$$

is switched on, since $\phi=x$ to leading order in $\delta$. The corresponding exponentially small correction to the height of the free surface is given by integrating with respect to $x$ as

$$
y_{\exp }=-2 \pi \mathrm{e}^{-\pi / \epsilon} \cos \left(\frac{x}{\epsilon}+\frac{\log a}{\epsilon}\right)
$$


Thus, the amplitude of gravity waves on the free surface is

$$
H \sim 2 \pi \delta \mathrm{e}^{-\pi / \epsilon},
$$

in agreement with (5.16).

\section{Appendix B. Canonical scaling}

We saw in the previous section that there is a distinguished limit when $\delta=O(\epsilon)$. This corresponds to both corners of the step being in the inner region of $\S$ A.1. In this section, we consider the beyond-all-orders analysis with this canonical scaling. The results here will bridge the gap between the order-one step analysis of $\S 5$, and the small-step analysis of Appendix A.

\section{B.1. Outer region}

We set $\delta=\beta \epsilon$. Then the outer problem is

$$
\begin{aligned}
\epsilon q^{2} \zeta \frac{\mathrm{d} q}{\mathrm{~d} \zeta} & =\sin \theta, \\
\log q & =\frac{1}{2} \log \left(\frac{\zeta+a+\epsilon \beta a}{\zeta+a}\right)-\frac{1}{\pi} \int_{0}^{\infty} \frac{\theta\left(\zeta^{\prime}\right) \mathrm{d} \zeta^{\prime}}{\zeta^{\prime}-\zeta} \\
& =\frac{1}{2} \log \left(1+\frac{\epsilon \beta a}{\zeta+a}\right)-\frac{1}{\pi} f_{0}^{\infty} \frac{\theta\left(\zeta^{\prime}\right) \mathrm{d} \zeta^{\prime}}{\zeta^{\prime}-\zeta} .
\end{aligned}
$$

As usual, we expand $q$ and $\theta$ in powers of $\epsilon$,

$$
q=\sum_{n=0}^{\infty} \epsilon^{n} q_{n}, \quad \theta=\sum_{n=0}^{\infty} \epsilon^{n} \theta_{n} .
$$

At leading order, we find $\theta_{0}=0, q_{0}=1$, whereas at $O(\epsilon)$ we find

$$
\theta_{1}=0, \quad q_{1}=\frac{\beta a}{2(\zeta+a)} .
$$

In general, we have

$$
\begin{gathered}
\zeta \frac{\mathrm{d} q_{n-1}}{\mathrm{~d} \zeta}+2 \zeta q_{1} \frac{\mathrm{d} q_{n-2}}{\mathrm{~d} \zeta}+\cdots=\theta_{n}+\cdots \quad(n \geqslant 3), \\
q_{n}-q_{n-1} q_{1}+\cdots-\mathrm{i} \theta_{n}=-\frac{(-1)^{n} \beta^{n} a^{n}}{2 n(\zeta+a)^{n}}-\frac{1}{\pi} \int_{-\infty}^{\infty} \frac{\theta_{n}\left(\zeta^{\prime}\right) \mathrm{d} \zeta^{\prime}}{\zeta^{\prime}-\zeta} \quad(n \geqslant 2) .
\end{gathered}
$$

As $n \rightarrow \infty$, we expect the asymptotic expansions to exhibit factorial/power divergence as before, so that we make the ansatz

$$
\theta_{n} \sim \frac{\Theta \Gamma(n+\gamma)}{\chi^{n+\gamma}}, \quad q_{n} \sim \frac{Q \Gamma(n+\gamma)}{\chi^{n+\gamma}} .
$$

The terms on the right-hand side of (B 6) are all exponentially small as $n \rightarrow \infty$ by comparison to those on the left. Thus we find,

$$
q_{n} \sim \mathrm{i} \theta_{n}+q_{n-1} q_{1}+\cdots,
$$

so that using (B 7) in (B 5) gives, to leading order as $n \rightarrow \infty$,

$$
\frac{\mathrm{d} \chi}{\mathrm{d} \zeta}=\frac{\mathrm{i}}{\zeta}
$$


Thus,

$$
\chi=\mathrm{i} \log (-\zeta / a),
$$

where we have used the fact that $\chi(-a)=0$. At the next order in $n$, we find

$$
\zeta \frac{\mathrm{d} Q}{\mathrm{~d} \zeta}-2 \zeta q_{1} \frac{\mathrm{d} \chi}{\mathrm{d} \zeta} Q=\mathrm{i} q_{1} Q,
$$

i.e.

so that

$$
\zeta \frac{\mathrm{d} Q}{\mathrm{~d} \zeta}=3 \mathrm{i} q_{1} Q=\frac{3 \mathrm{i} a \beta Q}{2(\zeta+a)},
$$

$$
Q=\Lambda\left(\frac{\zeta}{\zeta+a}\right)^{3 \mathrm{i} \beta / 2} \text {. }
$$

To determine $\gamma$ we need to examine the order of the singularity in $q_{n}$ as $\zeta \rightarrow-a$ to make sure that (B 7) is consistent with (B 4). As $\zeta \rightarrow-a$, we have

$$
q_{n} \sim \Lambda\left(\frac{-a}{\zeta+a}\right)^{3 \mathrm{i} \beta / 2} \frac{\Gamma(n+\gamma)}{(-\mathrm{i}(\zeta+a) / a)^{n+\gamma}} .
$$

For this to be consistent with (B 4), we must have $\gamma=-3 \mathrm{i} \beta / 2$. To determine $\Lambda$, we need to consider an inner region in the vicinity of the singularity at $\zeta=-a$.

\section{B.2. Inner region}

With the inner scaling $\zeta+a=\epsilon \mathrm{i} a z$ we have, at leading order,

$$
\begin{aligned}
\mathrm{i} q^{2} \frac{\mathrm{d} q}{\mathrm{~d} z} & =\sin \theta \\
q \mathrm{e}^{-\mathrm{i} \theta} & =\left(\frac{z-\mathrm{i} \beta}{z}\right)^{1 / 2} .
\end{aligned}
$$

Thus,

We let $\phi=q^{2}$, giving

$$
-2 q^{2} \frac{\mathrm{d} q}{\mathrm{~d} z}=q\left(\frac{z}{z-\mathrm{i} \beta}\right)^{1 / 2}-\frac{1}{q}\left(\frac{z-\mathrm{i} \beta}{z}\right)^{1 / 2} .
$$

We expand $\phi$ as $z \rightarrow \infty$ as

$$
-\phi \frac{\mathrm{d} \phi}{\mathrm{d} z}\left(1-\frac{\mathrm{i} \beta}{z}\right)^{1 / 2}=\phi-1+\frac{\mathrm{i} \beta}{z} .
$$

$$
\phi=\sum_{n=0}^{\infty} \frac{\phi_{n}}{z^{n}} .
$$

Then,

where

$$
\left(\sum_{n=0}^{\infty} \frac{\phi_{n}}{z^{n}}\right)\left(\sum_{n=1}^{\infty} \frac{n \phi_{n}}{z^{n+1}}\right)\left(\sum_{n=0}^{\infty} \frac{c_{n}}{z^{n}}\right)=\sum_{n=0}^{\infty} \frac{\phi_{n}}{z^{n}}-1+\frac{\mathrm{i} \beta}{z},
$$

so that

$$
c_{n}=\frac{\Gamma(3 / 2)(-\mathrm{i} \beta)^{n}}{\Gamma(3 / 2-n) n !},
$$

$$
\phi_{0}=1 \text {, }
$$




$$
\begin{aligned}
& \phi_{1}=-\mathrm{i} \beta \\
& \phi_{n}=\sum_{m=0}^{n-1} \sum_{k=0}^{n-m-1} k \phi_{m} \phi_{k} c_{n-m-k-1} \quad(n \geqslant 2) .
\end{aligned}
$$

Now the outer limit of the $n$th term in the inner expansion of $q^{2}$

$$
\sim \frac{\phi_{n}}{z^{n}}
$$

while the inner limit of the $n$th term in the outer expansion of $q^{2}$

$$
\sim 2 \epsilon^{n} q_{0} q_{n} \sim 2 \Lambda \mathrm{e}^{-3 \pi \beta / 4} \frac{\Gamma(n-3 \mathrm{i} \beta / 2)}{z^{n}} .
$$

Matching gives

$$
\Lambda=\frac{\mathrm{e}^{3 \pi \beta / 4}}{2} \lim _{n \rightarrow \infty} \frac{\phi_{n}}{\Gamma(n-3 \mathrm{i} \beta / 2)} .
$$

\section{B.3. Stokes lines and gravity waves}

There is a Stokes line where $\chi$ is real and positive, i.e. where $|\zeta|=a$, or $\operatorname{Re}(w)=-\log a$. Thus, there is a Stokes line which cuts the real free boundary at $\phi=-\log a$.

An analysis identical to that of $\S 4$ shows that across this Stokes line, the exponentially small correction term

$$
\begin{aligned}
\theta_{\exp } & \sim \frac{2 \pi \mathrm{i}}{\epsilon^{\gamma}} \Theta \mathrm{e}^{-\chi / \epsilon}+\text { complex conjugate } \\
& =2 \pi \Lambda\left(\frac{\epsilon \zeta}{\zeta+a}\right)^{3 \mathrm{i} \beta / 2} \mathrm{e}^{-\pi / \epsilon} \mathrm{e}^{-\mathrm{i} \log (\zeta / a) / \epsilon}+\text { complex conjugate } \\
& =4 \pi|\Lambda| \mathrm{e}^{-\pi / \epsilon} \cos \left(-\frac{\log (\zeta / a)}{\epsilon}+\arg (\Lambda)+\frac{3 \beta}{2} \log \left(\frac{\epsilon \zeta}{\zeta+a}\right)\right) \\
& =4 \pi|\Lambda| \mathrm{e}^{-\pi / \epsilon} \cos \left(\frac{\phi}{\epsilon}+\frac{\log a}{\epsilon}+\arg (\Lambda)-\frac{3 \beta \phi}{2}+\frac{3 \beta}{2} \log \left(\frac{\epsilon}{\mathrm{e}^{-\phi}+a}\right)\right)
\end{aligned}
$$

is switched on. Since $\phi=x$ to leading order, the corresponding exponentially small correction to the height of the free surface is given by

$$
y_{\exp } \sim 4 \pi|\Lambda| \epsilon \mathrm{e}^{-\pi / \epsilon} \sin \left(\frac{\phi}{\epsilon}+\frac{\log a}{\epsilon}+\arg (\Lambda)-\frac{3 \beta \phi}{2}+\frac{3 \beta}{2} \log \left(\frac{\epsilon}{\mathrm{e}^{-\phi}+a}\right)\right) .
$$

Thus, the amplitude of the gravity waves at infinity in this case is

$$
H \sim 4 \pi|\Lambda| \epsilon \mathrm{e}^{-\pi / \epsilon} .
$$

This result provides a smooth transition between the $\delta=O(1)$ case considered in $\S 5$ and the $\delta \ll \epsilon$ case considered in Appendix A.

Let us check that (B 16) matches with (5.13) as $\beta \rightarrow \infty$ and with (5.16) as $\beta \rightarrow 0$. To match with (5.16) we require that $|\Lambda(\beta)| \sim \beta / 2$ as $\beta \rightarrow 0$; figure 9 shows that this is indeed the case.

The $b=O(1)$ result (5.13) written in terms of $\beta$ is

$$
H \sim \frac{0.82}{\epsilon^{1 / 5}}(\beta \epsilon)^{6 / 5} \exp \left(-\frac{\pi}{\epsilon}\left(\frac{1}{1+\beta \epsilon}\right)^{3 / 2}\right) \sim 0.82 \epsilon \beta^{6 / 5} \exp \left(-\frac{\pi}{\epsilon}+\frac{3 \beta \pi}{2}\right) .
$$




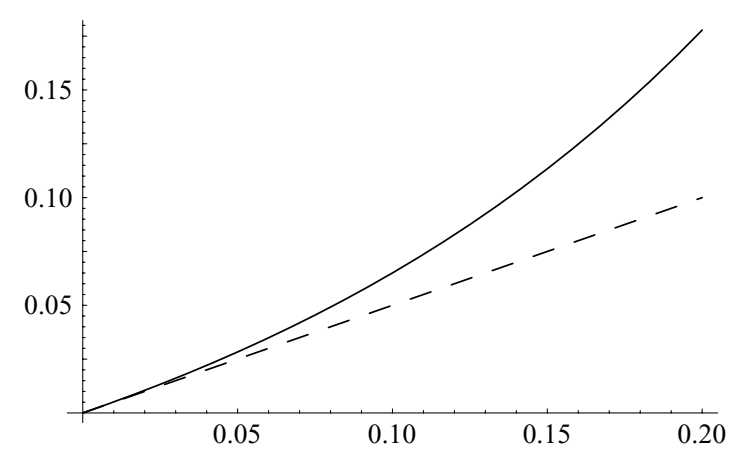

FIgURE 9. The function $|\Lambda(\beta)|$ (solid curve). As $\beta \rightarrow 0,|\Lambda| \sim \beta / 2$ (dashed curve).

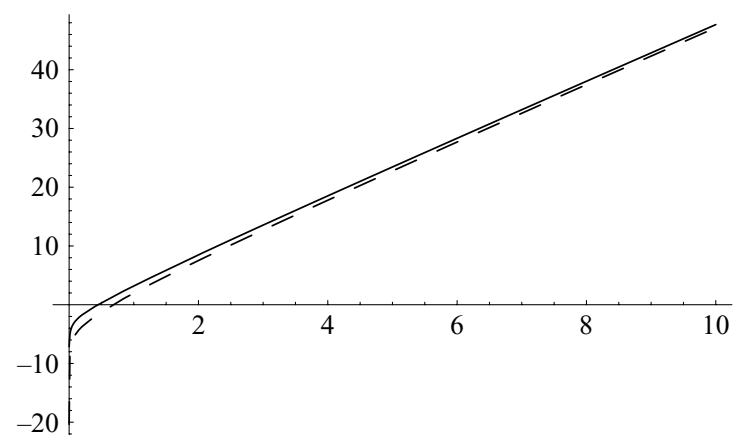

FIGURE 10. The natural logarithm of $|\Lambda(\beta)|$ (solid curve). As $\beta \rightarrow \infty$, $|\Lambda| \sim 0.82 /(4 \pi) \beta^{6 / 5} \mathrm{e}^{3 \beta \pi / 2}$. The natural logarithm of this function is shown as a dashed curve.

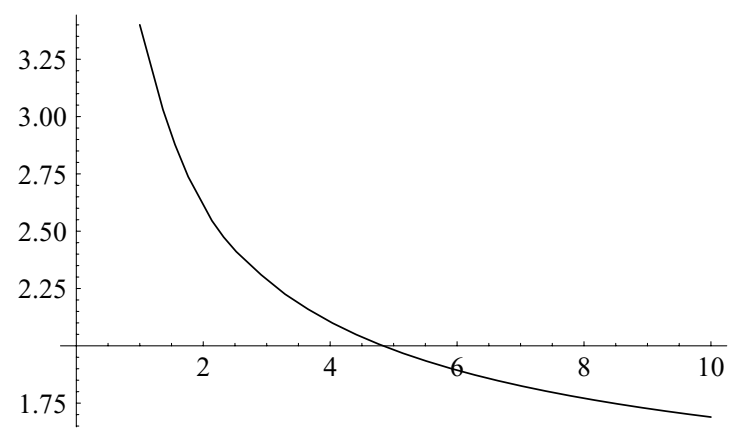

FIgURE 11. The function $|\Lambda(\beta)|$ divided by its asymptotic limit $0.82 /(4 \pi) \beta^{6 / 5} \mathrm{e}^{3 \beta \pi / 2}$. As $\beta \rightarrow \infty$ the curve should tend to unity.

For this to match with (B 16) we require that

$$
\Lambda(\beta) \sim \frac{0.82}{4 \pi} \beta^{6 / 5} \mathrm{e}^{3 \beta \pi / 2}
$$

as $\beta \rightarrow \infty$. The matching is illustrated in figures 10 and 11 . We note that the convergence as $\beta \rightarrow \infty$ is slow. This explains why, as we saw in $\S 6$, a uniform approximation generated from the $b=O(1)$ and $b=O(\epsilon)$ results does much better than the $b=O(1)$ approximation for moderate Froude numbers. 


\section{REFERENCES}

Akylas, T. \& YAng, T.-S. 1995 On short-scale oscillatory tails of long-wave disturbances. Stud. Appl. Maths 94, 1-20.

Belward, S. R. \& Forbes, L. K. 1993 Fully nonlinear two-layer flow over arbitrary topography. J. Engng Maths 27, 419-432.

Berry, M. V. 1989 Uniform asymptotic smoothing of Stokes discontinuities. Proc. R. Soc. Lond. A 422, 7-21.

ByatT-Smith, J. 1991 On the existence of homoclinic and heteroclinic orbits for differential equations with a small parameter. Eur. J. Appl. Maths 2, 133-158.

Chapman, S. J. 1999 On the rôle of Stokes lines in the selection of Saffman-Taylor fingers with small surface tension. Eur. J. Appl. Maths 10, 513-534.

Chapman, S. J. \& King, J. R. 2003 The selection of Saffman-Taylor fingers by kinetic undercooling. J. Engng Maths 46, 1-32.

Chapman, S. J. \& Vanden-Broeck, J.-M. 2002 Exponential asymptotics and capillary waves. SIAM J. Appl. Maths 62, 1872-1898.

Chapman, S. J., King, J. R. \& Adams, K. L. 1998 Exponential asymptotics and Stokes lines in nonlinear ordinary differential equations. Proc. R. Soc. Lond. A 454, 2733-2755.

Combescot, R., Hakim, V., Dombre, T., Pomeau, Y. \& Pumir, A. 1988 Analytic theory of the Saffman-Taylor fingers. Phys. Rev. A 37, 1270-1283.

DEAN, W. R. 1948 On the reflection of surface waves by a submerged circular cylinder. Proc. Camb. Phil. Soc. 44, 483-491.

Dias, F. \& VANDEn-Broeck, J.-M. 2004 Trapped waves between submerged obstacles. J. Fluid Mech. 509, 93-102.

Dingle, R. B. 1973 Asymptotic Expansions: their Derivation and Interpretation. Academic.

Forbes, L. K. \& Hocking, G. C. 1990 Flow caused by a point sink in a fluid having a free surface. J. Austral. Math. Soc. B 32, 231-249.

Forbes, L. K. \& Schwartz, L. W. 1982 Free surface flow over a semi-circular obstruction. J. Fluid Mech. 114, 299-314.

GAZDAR, A. S. 1973 Generation of waves of small amplitude by an obstacle placed on the bottom of a running stream. J. Phys. Soc. Japan 34, 530.

GrimshaW, R. \& Joshi, N. 1995 Weakly nonlocal solitary waves in a singularly perturbed Kortewegde Vries equation. SIAM J. Appl. Maths 55, 124-135.

HAкIM, V. 1991 Computation of transcendental effects in growth problems: linear solvability conditions and nonlinear methods. In Asymptotics Beyond all Orders (ed. H. Segur, S. Tanveer \& H. Levine), NATO ASI Series B, vol. 284, pp. 15-28. Plenum.

Havelock, T. H. 1927 The method of images in some problems of surface waves. Proc. R. Soc. Lond. A 15, 268-280.

Kelvin, L. 1886 On stationary waves in flowing water. Phil. Mag. 22, 445.

von KerczeK, C. \& Salvesen, N. 1977 Nonlinear free surface effects - the dependence on Froude number. In Proc. 2nd Intl Conf. on Numerical Ship Hydrodynamics, Berkeley, p. 202.

King, A. C. \& Bloor, M. I. G. 1987 Free-surface flow over a step. J. Fluid Mech. 182, 193-208.

KING, A. C. \& BLooR, M. I. G. 1989 A semi-inverse method for free-surface flow over a submerged body. Q. J. Mech. Appl. Maths 42, 183-202.

King, A. C. \& BLoOR, M. I. G. 1990 Free-surface flow of a stream obstructed by an arbitrary bed topography. Q. J. Mech. Appl. Maths 43, 87-106.

Koerber, A. J. \& Forbes, L. K. 1998 Two-dimensional steady free surface flow into a semi-infinite mat sink. Phys. Fluids 10, 2781-2785.

Koerber, A. J. \& Forbes, L. K. 1999 Cusp flows due to an extended sink in two dimensions. J. Engng Maths 36, 349-360.

Kruskal, M. D. \& Segur, H. 1991 Asymptotics beyond all orders in a model of crystal growth. Stud. Appl. Maths 85, 129-181.

Lamb, H. 1913 On some cases of wave motion on deep water. Ann. Mat. 21, 237.

Pomeau, Y., Ramani, A. \& Grammaticos, B. 1988 Structural stability of the Korteweg-de Vries solitons under a singular perturbation. Physica D 31, 127-134.

SCullen, D. \& TuCK, E. O. 1995 Nonlinear free-surface flow computations for submerged cylinders. J. Ship Res. 39, 185-193. 
TuCK, E. O. \& SCUlleN, D. C. 2002 A comparison of linear and nonlinear computations of waves made by slender submerged bodies. J. Engng Maths 42, 255-264.

Tuck, E. O. \& VANDEN-Broeck, J.-M. 1984 A cusp-like free-surface flow due to a submerged source or sink. J. Austral. Math. Soc. B 25, 443-450.

Tuck, E. O. \& Vanden-Broeck, J.-M. 1998 Ploughing flows. Eur. J. Appl. Maths 9, 463-483.

URSELl, F. 1950 Surface waves on deep water in the presence of a submerged circular cylinder. Proc. Camb. Phil. Soc. 46, 141-158.

Vanden-Broeck, J.-M. \& Keller, J. B. 1997 An axisymmetric free surface with a 120 degree angle along a circle. J. Fluid Mech. 342, 403-409.

WiLmotт, P. 1987 On the motion of a small two-dimensional body submerged beneath surface waves. J. Fluid Mech. 176, 465-481. 